2018-10

\title{
A multi-envelope vertical coordinate system for numerical ocean modelling
}

\author{
Bruciaferri, Diego
}

http://hdl.handle.net/10026.1/11788

10.1007/s10236-018-1189-x

Ocean Dynamics

Springer Verlag

All content in PEARL is protected by copyright law. Author manuscripts are made available in accordance with publisher policies. Please cite only the published version using the details provided on the item record or document. In the absence of an open licence (e.g. Creative Commons), permissions for further reuse of content should be sought from the publisher or author. 


\title{
A multi-envelope vertical coordinate system for numerical ocean modelling
}

\author{
Diego Bruciaferri • Georgy I. Shapiro • \\ Fred Wobus
}

Received: 5 January 2018 / Accepted: 19 June 2018

Please cite as:

Bruciaferri, D., Shapiro, G.I. \& Wobus, F. Ocean Dynamics (2018). https://doi.org/10.1007/s10236-018-1189-x

\begin{abstract}
A Multi-Envelope generalised coordinate system for numerical ocean modelling is introduced. In this system, computational levels are curved and adjusted to multiple 'virtual bottoms' (aka envelopes) rather than following geopotential levels or the actual bathymetry. This allows defining computational levels which are optimised to best represent different physical processes in different subdomains of the model. In particular, we show how it can be used to improve the representation of tracer advection in the ocean interior. The new vertical system is compared with a widely used z-partial step scheme. The modelling skill of the models is assessed by comparison with the analytical solutions or results produced by a model with a very high resolution z-level grid. Three idealised process-oriented numerical experiments are carried out. Experiments show that numerical errors produced by the new scheme are much smaller than those produced by the standard z-partial step scheme at a comparable vertical resolution. In particular, the new scheme shows superiority in simulating the formation of a cold intermediate
\end{abstract}

D. Bruciaferri

Faculty of Science and Engineering,

University of Plymouth, PL4 8AA, UK

E-mail: diego.bruciaferri@plymouth.ac.uk

G. I. Shapiro

Faculty of Science and Engineering,

University of Plymouth, PL4 8AA, UK

Shirshov Institute of Oceanology

36 Nahimovski prospect, Moscow, 117997, Russia

F. Wobus

Faculty of Science and Engineering,

University of Plymouth, PL4 8AA, UK 
layer in the ocean interior and in representing dense water cascading down a steep topography.

Keywords Ocean modelling · Vertical coordinate · Oceanic transport

\section{$1 \quad 1$ Introduction}

2 When designing an ocean model, the choice of the vertical coordinate system must be pursued very carefully (Griffies, 2004), especially in regional areas where local smaller-scale processes interact with large-scale oceanographic features (Kantha and Clayson, 2000; Gangopadhyay and Robinson, 2002). Numerical discretisation introduces truncation errors specific to the chosen vertical coordinate system, and hence influences the representation of physical processes (Haidvogel and Beckmann, 1999). Currently, three main vertical coordinates are typically used in ocean modelling, namely $z$-level (geopotential), terrain-following and isopycnic, but all of them have deficiencies (see e.g Chassignet et al. 2006).

The $z$-level coordinates are a natural framework for describing horizontal pressure gradients. However, the $z$-level system generates an unnatural step-like representation of bottom topography and consequently introduces an error in simulating near-bottom processes, including dense water overflows (e.g. Ezer and Mellor 2004; Ivanov et al. 2004). Gerdes (1993a) concluded that the crude approximation to the actual topography used in $z$-level models results in large errors in the simulated mass transport in regions where planetary and topographic beta-effects are of comparable magnitudes. Horizontal overshoots of dense water due to step-like representation of bottom topography lead to spurious convective mixing. Recently, Ezer (2016) showed that the unrealistic representation of topographic slopes in $z$ ocean models has a negative impact on the simulation of the dynamics of western boundary currents and consequently of large-scale circulation.

The disadvantages of $z$-level grids initiated intensive development of terrainfollowing grids for ocean modelling (Blumberg and Mellor, 1987; Haidvogel et al., 1991; Ezer and Mellor, 1992). The terrain-following coordinate ( $\sigma$-coordinate system) offers a smooth representation of bottom topography and a natural parametrisation of the bottom boundary layer (Mellor et al., 2002). However, it introduces a pressure gradient error, in particular on steep slopes (Haney, 1991; Mellor et al., 1994, 1998).

The use of computational surfaces that are not aligned with isopycnals (which is generally the case of both $z$-and $\sigma$-coordinate systems) in simulating tracer transport introduces the contamination of slow diapycnal processes by fast isopycnal exchanges (e.g. Roberts and Marshall 1998; Griffies et al. 2000b). As a consequence, spurious diapycnic mixing poses a major problem in non-isopycnal models (see Holt et al. 2017 and references therein). Such deficiencies are not present in vertical grids where computational levels follow isopycnals (so-called isopycnic grids), e.g. used in the MICOM ocean model, see Bleck (1998). However, isopycnic models experience difficulties in weakly stratified areas, such as over the continental shelf or in the upper or bottom mixed layers (Griffies et al., 2000a). Legg et al. (2006) compared the performance of isopycnal and $z$-models in representing dense cascades while Legg et al. (2009) pointed out the importance of a correct simulation of oceanic overflows in numerical climate models. 
In order to minimise the disadvantages of the various vertical coordinate systems, further modifications were introduced either to the vertical grids themselves or to the numerical representation of the governing equations. For example, the introduction of shaved (Adcroft et al., 1997) or partial (Pacanowski et al., 1998) cells which slightly change the shape of 'pure' $z$-coordinate grids was proposed to improve the representation of bottom topography in $z$-models. The $z$-partial steps approach is now widely used for global (Barnier et al., 2006) and regional (e.g., Oddo et al. 2009; Trotta et al. 2016) ocean models. A stretched terrain-following $s$ coordinate system(Song and Haidvogel, 1994) and its several variants (e.g, Madec et al. 1996; Siddorn and Furner 2013) as well as advanced methods in calculation of pressure gradients (Shchepetkin, 2003) were developed to improve $\sigma$-coordinates flexibility and accuracy.

The concept of a generalised vertical coordinate system (see for example Kasahara 1974 or Mellor et al. 2002) allowed in principle the development of vertical grids of various complexity, as for example the hybrid vertical schemes where different 'pure' grids were applied to different sub-domains of the ocean. The aim of this was to better represent the differing physical processes which might prevail in different sub-domains, by using one specific grid rather than another. Examples of those methods are the HYCOM model (Bleck and Boudra, 1981; Bleck, 2002), the vertical grids by Gerdes (1993a,b), Madec et al. (1996), Shapiro et al. (2013) or the Song and Hou (2006) parametric vertical coordinate system.

The idea of Arbitrary Lagrangian-Eulerian (ALE) vertical coordinates (Hirt et al., 1974) permitted the development of $z^{*}$ - (Adcroft and Campin, 2004) and $\tilde{z}$-coordinates (Leclair and Madec, 2011) and the adaptive $\sigma$-based coordinate (Hofmeister et al., 2010).

A significant improvement in terrain-following schemes was achieved by introducing the idea of the 'enveloping' bathymetry, where computational surfaces follow a 'virtual bottom' (aka envelope) rather than the real bathymetry (Enriquez et al., 2005; Dukhovskoy et al., 2009; Shapiro et al., 2013). This solution allows the reduction of slopes of computational surfaces and the reduction of pressure gradient errors to an acceptable level.

In this study, we introduce the 'Multi-Envelope $s$-coordinate' (hereinafter MEscoordinate). It extends the classical concept of terrain-following coordinates by defining $s$-levels which follow multiple envelopes rather than a single one as is the case in existing models. This approach allows to combine the ideas behind the hybrid schemes (best representation of different physics in different sub-domains of the model) and numerical improvements (e.g. enveloping) developed for 'pure' vertical discretisation grids. The new vertical system represents a generalised coordinate system, since all non-isopycnal vertical grids (both 'pure' and hybrid) can be considered a special case of MEs-coordinates.

The paper is organized as follows. Section 2 defines the MEs-coordinate, detailing its features. Section 3 describes the idealised model domain, the design of the three different vertical grids and the set up of the three numerical experiments. In Section 4, the results are presented, analysed and discussed. Section 5 summarises our main conclusions. 


\section{The Multi-Envelope $s$-coordinate}

In this paper we show how the MEs system can be used to improve the representation of the oceanic transport in a non-isopycnal coordinate model. The MEs-coordinate combines the $s$-coordinate concept and the idea of 'enveloping' the bottom topography.

Let us consider a local Cartesian $x, y, z$ coordinate system with a downward vertical unit vector $\hat{z}$. A stretched envelope-following $s$-coordinate can be defined as

$$
z=S\left(\sigma, \eta, H_{e}\right)
$$

where $\eta(x, y, t)$ is the deviation of the sea surface from its unperturbed position, $H_{e}(x, y)$ is a smoothed version of the actual bottom topography (aka bathymetry envelope) and $-1 \leq \sigma \leq 0$, with $\sigma=0$ at $z=\eta$ and $\sigma=-1$ at $z=H_{e}$. A general stretching function is represented by $S\left(\sigma, \eta, H_{e}\right)$. It can be, for example, the one by Song and Haidvogel (1994), Shchepetkin and McWilliams (2005) or Siddorn and Furner (2013).

The MEs vertical system defines $n$ arbitrary reference surfaces (hereafter called envelopes) $H_{e}^{k}(x, y, t)$, with $0 \leq k \leq n$ and $n \in\{2 m+1\}$ with $m$ a positive integer such that

$$
\eta=H_{e}^{0}<H_{e}^{1}<\ldots<H_{e}^{n-1}<H_{e}^{n}
$$

Each envelope $H_{e}^{k}(x, y, t)$ moves with the free-surface according to the following equation:

$$
H_{e}^{k}=h_{e}^{k}+\eta\left(1-\frac{h_{e}^{k}}{h}\right)
$$

where $h_{e}^{k}(x, y)$ is the depth of the $k^{t h}$ envelope when the ocean free-surface is unperturbed $(\eta=0)$ and $h=h_{e}^{n}$.

The envelopes divide the ocean model vertical domain into $n$ sub-zones $D_{i}$, with $1 \leq i \leq n$. Each sub-zone $D_{i}$ is bounded by envelopes $H_{e}^{i-1}$ at the top and $H_{e}^{i}$ at the bottom.

The non-dimensional $\sigma_{i}$-coordinate is defined for each sub-zone $D_{i}$ as

$$
\sigma_{i}=-\frac{z-H_{e}^{i-1}}{H_{e}^{i}-H_{e}^{i-1}}
$$

with $\sigma_{i}\left(H_{e}^{i-1}\right)=0$ and $\sigma_{i}\left(H_{e}^{i}\right)=-1$. Then, the MEs-coordinate is defined as a piecewise function

$$
\begin{cases}\left.z\right|_{D_{i}}=S_{i}\left(\sigma_{i}, H_{e}^{i-1}, H_{e}^{i}\right), & \text { if } i \in\{2 m+1\} \\ \left.z\left(x, y, \sigma_{i}, t\right)\right|_{D_{i}}=P_{x, y, i}^{3}\left(\sigma_{i}\right), & \text { if } i \in\{2 m\}\end{cases}
$$

The function $S_{i}\left(\sigma_{i}, H_{e}^{i-1}, H_{e}^{i}\right)$ in Equation 5a represents a general stretching function. For example, in the case of the classical Song and Haidvogel (1994) stretching function, MEs coordinates are defined as

$$
\left.z\right|_{D_{i}}=H_{e}^{i-1}+h_{c}^{i} \sigma_{i}-C_{i}\left(\sigma_{i}\right)\left(H_{e}^{i}-h_{c}^{i}-H_{e}^{i-1}\right)
$$

where $h_{c}^{i}$ is the critical depth at which transition from pure $\sigma$ to the stretched $s$-coordinate occurs and $C_{i}\left(\sigma_{i}\right)$ is the hyperbolic function of Song and Haidvogel (1994) (their $C(s)$ ).

The function $P_{x, y, i}^{3}\left(\sigma_{i}\right)$ in Equation $5 \mathrm{~b}$ is a complete cubic spline whose coefficients are determined by the following three constraints: 


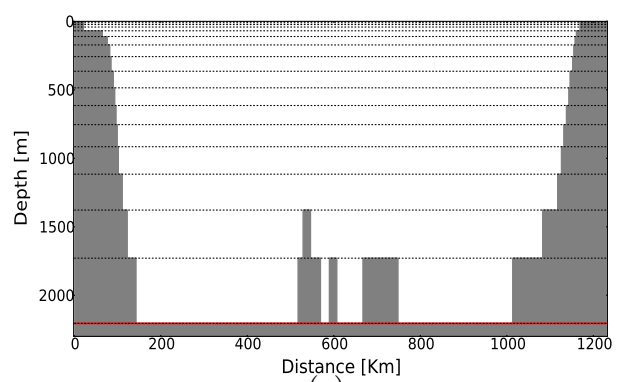

(a)

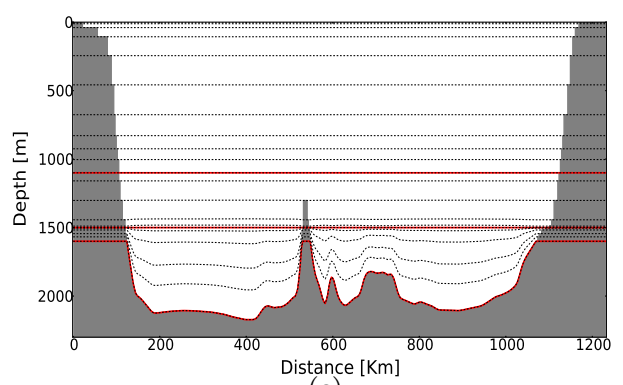

(c)

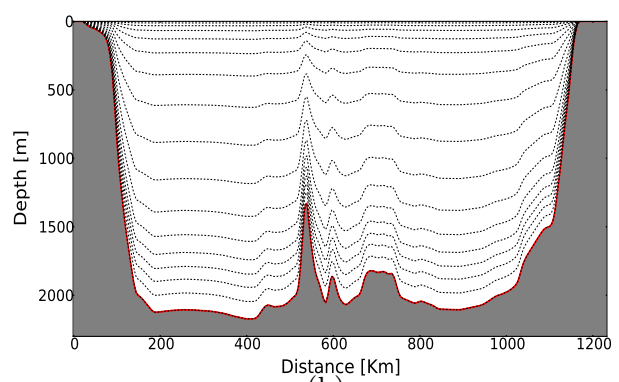

(b)

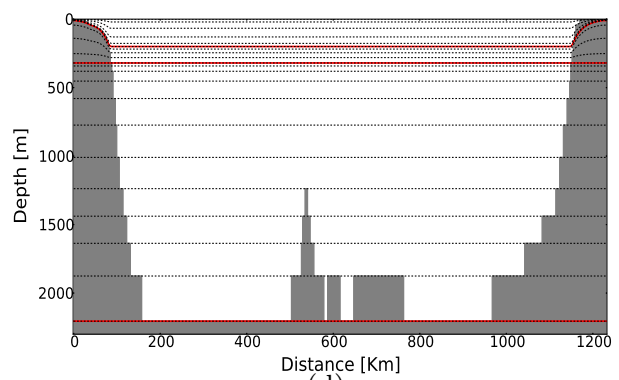

(d)

Fig. 1 Sketches depicting 'pure' $z$ - (a) and $\sigma$ - (b) grids and hybrid Madec et al. (1996) $z$ on-top-of-s (c) and Shapiro et al. (2013) $s$-on-top-of- $z$ (d) approaches as retrieved with the MEs-coordinate system. Envelopes $H_{e}^{i}$ used to define each specific configuration are shown in red.

1. Monotonicity:

$$
\begin{gathered}
\left.\partial_{\sigma_{i}} z\right|_{D_{i}}>0, \text { with } \\
\begin{cases}-1 \leq \sigma_{i} \leq 0, & \text { if } i=n \\
-1<\sigma_{i} \leq 0, & \text { if } i<n\end{cases}
\end{gathered}
$$

2. Continuity:

$$
\left.z\right|_{D_{i}}\left(\sigma_{i}=-1\right)=\left.z\right|_{D_{i+1}}\left(\sigma_{i+1}=0\right)
$$

3. Continuity of the first derivative:

$$
\left.\partial_{\sigma_{i}} z\right|_{D_{i}}\left(\sigma_{i}=-1\right)=\left.\partial_{\sigma_{i+1}} z\right|_{D_{i+1}}\left(\sigma_{i+1}=0\right)
$$

A description of the method used to determine the coefficients of complete cubic splines $P_{x, y, i}^{3}\left(\sigma_{i}\right)$ is given in Appendix 1. Under these conditions, the Jacobian of the transformation from $z$ to $\sigma$ is continuous, ensuring one of the requirements of improved accuracy formulated by Marti et al. (1992) and Treguier et al. (1996).

The new MEs represents a generalised coordinate system, in the sense that 'pure' and hybrid non-isopycnal vertical coordinates can be considered a special case of MEs-coordinate. For example, $z$-grids are simply generated by defining a single horizontal envelope $H_{e}^{1}=\max \left(H_{B}\right)$, where $H_{B}(x, y)$ is the actual bathymetry (see Figure 1(a)). Similarly, terrain-following $\sigma$-coordinates can be 
generated by choosing $H_{e}^{1}=H_{B}$, see Figure 1(b)). Figures 1(c) and 1(d) show how hybrid ' $z$-on-top-of-s' (Madec et al., 1996) and ' $s$-on-top-of- $z$ ' (Shapiro et al., 2013) grids, respectively, can be easily generated with the MEs vertical system. In MEs all grid cells are full, both near the bottom and in the interior, and their shape is dictated by the corresponding envelope.

An important feature of the MEs system is that envelopes $H_{e}^{i}$ can be arbitrarily chosen surfaces. This implies that they can be designed to optimise the representation of those physical processes that are prioritised, allowing the modeller to manage and control the design of model levels with enhanced flexibility. Figure 2 shows an example of MEs design by using five reference surfaces $H_{e}^{i}$.

In this configuration, sub-zone $D_{5}$ has model levels which follow envelope $H_{e}^{5}$, a smooth version of the actual bottom topography up to $1500 \mathrm{~m}$. This enables realistic simulations of dense water overflows over the ocean bottom while reducing pressure gradient errors. In sub-zone $D_{3}$, model levels are horizontal. Zones $D_{2}$ and $D_{4}$ work as transition zones which gradually reduce the slope of $s$-levels towards geopotential surfaces in $D_{3}$.

The upper envelope $H_{e}^{1}$ follows the 'main pycnocline' (i.e. long-term mean pycnocline) in open ocean areas but it follows the topography in coastal regions. Such an envelope allows to obtain realistic simulations of both dense water cascades in shelf areas and the formation of a cold intermediate layer in the open sea. The pycnocline-shaped envelope reduces the angle between the computational surfaces and the isopycnals, and hence reduces the spurious diapycnal mixing, thus performing similar to isopycnal coordinate systems.

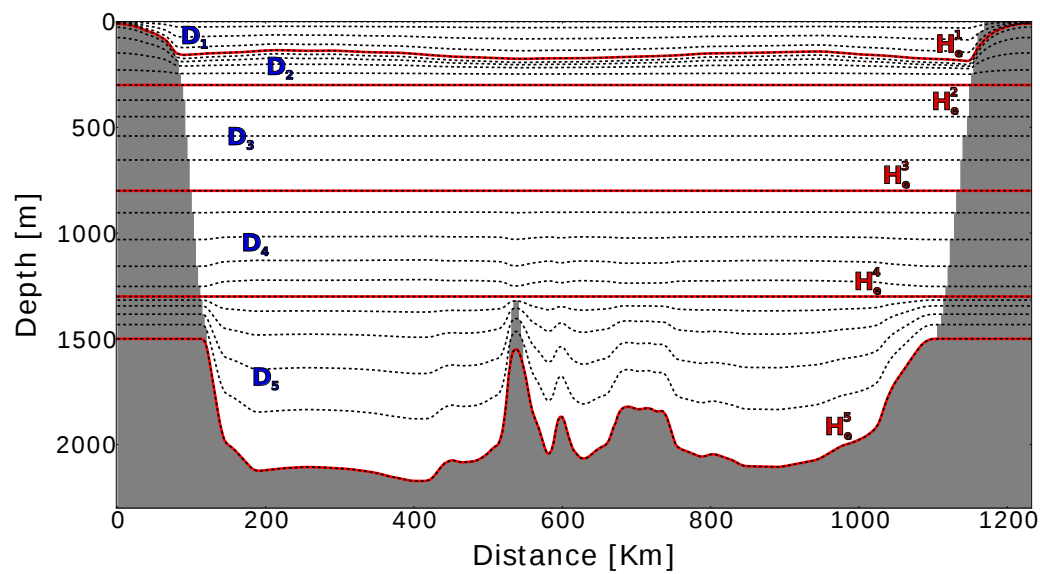

Fig. 2 Conceptual sketch of the MEs vertical coordinate system. In this example, five envelopes $H_{e}^{i}$ are used to define MEs-levels.

To clarify this effect, let us consider the idealised case of a two-layer immiscible fluid depicted in Figure 3.

In this case, tracer advection and diffusion occurs exclusively along the isopycnal surface, as represented by black and green arrows in Figure 3(a), and there is 


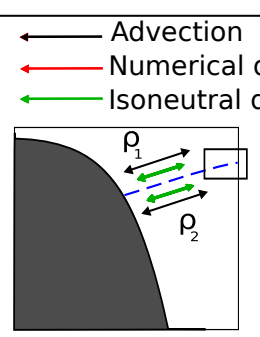

(a)

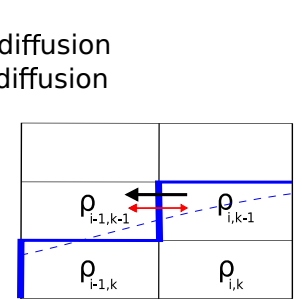

(b)

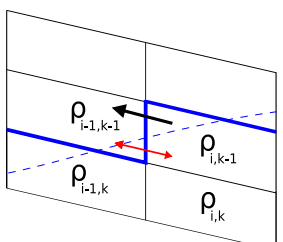

(c)

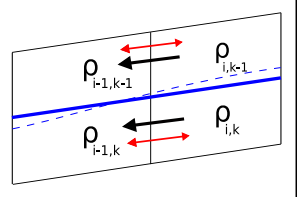

(d)

Fig. 3 Idealised two density layers baroclinic ocean (a) and its representation with geopotential $z$-levels (b), terrain-following $s$-levels (c) and the MEs vertical system with the upper envelope $H_{e}^{1}$ designed to follow the main pycnocline in open ocean areas (d). The real pycnocline is represented by the dashed blue lines, while the pycnocline simulated by the models is shown with the solid blue lines. See the text for more detailed explanations.

no diapycnal mixing. Figures 3(b), 3(c) and 3(d) illustrate how the real isopycnal surface is represented with $z$-level, $s$-level and MEs grids, respectively.

Black arrows in Figures 3(b) and 3(c) show how advection is simulated in $z$ and $s$-models, resulting in the spurious mixing across different densities due to much stronger 'along-computational-level' numerical diffusion (see the red arrows) , which transfers mass and momentum between the density layers.

The rotation of the diffusion operator to align the lateral diffusion with isopycnals (Redi, 1982) would have reduced this undesirable effect. However, s-models typically use geopotentially oriented diffusion, because of the difficulties in computing isoneutral diffusion (Barnier et al., 1998; Marchesiello et al., 2009; Furner, 2012; Lemarié et al., 2012). Another approach (widely used in regional models) could be the subtraction of climatological temperature and salinity fields before the lateral diffusion fluxes are calculated, hence diffusing only tracer anomalies, following Mellor and Blumberg (1985).

If model levels mimic the pycnocline as in the MEs model, the angle between the isopycnals and computational surfaces is small, see Figure 3(c), and the spurious diapycnal mixing arising from numerical errors of the advective schemes is significantly reduced.

\section{Experiments to assess model skill}

In this section we assess the modelling skills of the MEs scheme in comparison to the widely used $z$-level with partial steps scheme by performing a set of idealised numerical experiments with an axisymmetric ocean basin.

The model domain is a bowl-shape basin with a diameter of $500 \mathrm{~km}$, maximum depth of $1000 \mathrm{~m}$ and the downward positive topography $H_{B}$ defined by

$$
H_{B}=\max \left\{h_{0} \exp \left(\frac{x^{2}}{2 \sigma^{2}}+\frac{y^{2}}{2 \sigma^{2}}\right), 1000\right\}
$$

with $h_{0}=25000 \mathrm{~m}, \sigma=8$, and $x, y \in[-40 \mathrm{~km}, 40 \mathrm{~km}]$ (see Figure 4(a)). The slope at the $200 \mathrm{~m}$ isobath of the idealised basin is $\approx 1.5 \%$. 


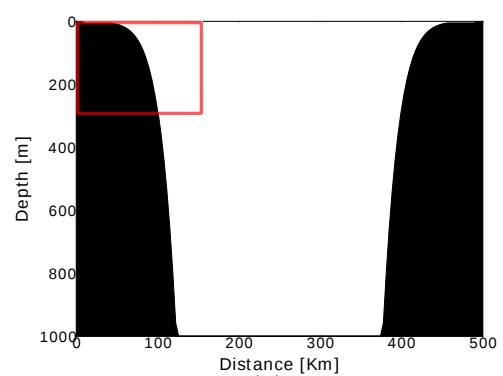

(a)

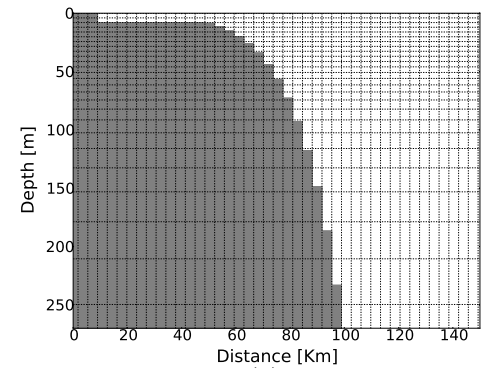

(c)

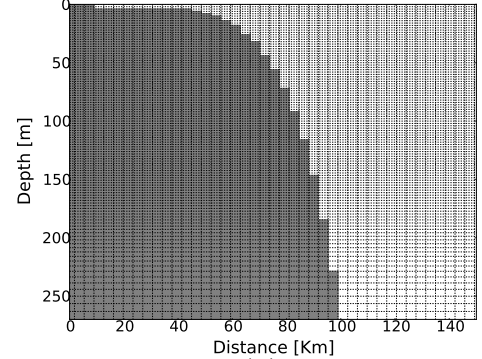

(b)

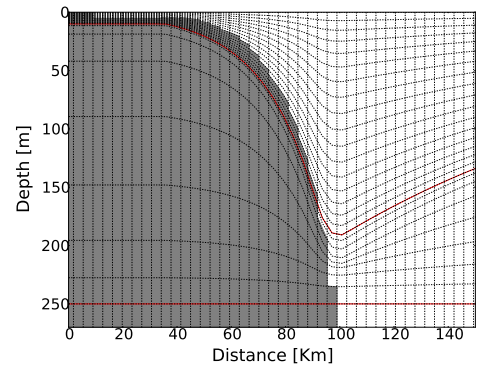

(d)

Fig. 4 Cross sections of the topography $H_{B}$ of the idealised domain (a) and $z f s-150$ (b), zps-34 (c) and MEs-34 vertical (d) grids configured for this study. For the numerical grids, only the portion of the domain highlighted with the red square in panel (a) is shown for clarity.

In order to use the MEs grid for our computations, we modified the Nucleus for European Modelling of the Ocean (NEMO) Ocean General Circulation model code accordingly. The NEMO hydrodynamic component is a three dimensional, finite differences, free-surface primitive equation ocean model suitable for modelling ocean circulation at regional and global scales. It solves the incompressible, hydrostatic, Boussinesq approximated primitive equations along with a non-linear equation of state. NEMO provides a selection of various turbulence closure schemes. In this study we use the NEMO 3.6-stable code, see Madec (2008).

\subsection{Model grids}

All the numerical experiments are carried out by using two models which have the same horizontal mesh but two different vertical grids: one uses the common $z$-level with partial steps (hereafter called $z p s$ ) while the second uses the new MEs scheme. In the horizontal, the mesh has 140 grid points in both the zonal and the meridional directions and a uniform grid spacing $\Delta x=\Delta y \approx 3.57 \mathrm{~km}$. For better comparison between the MEs against the zps vertical grid, both models have the same number of 34 numerical levels and hereafter they are called MEs-34 and $z p s-34$, respectively. For the same reason, the computational level $\mathrm{n}^{o} 26$ is placed at the same depth of $250 \mathrm{~m}$ for both grids. 


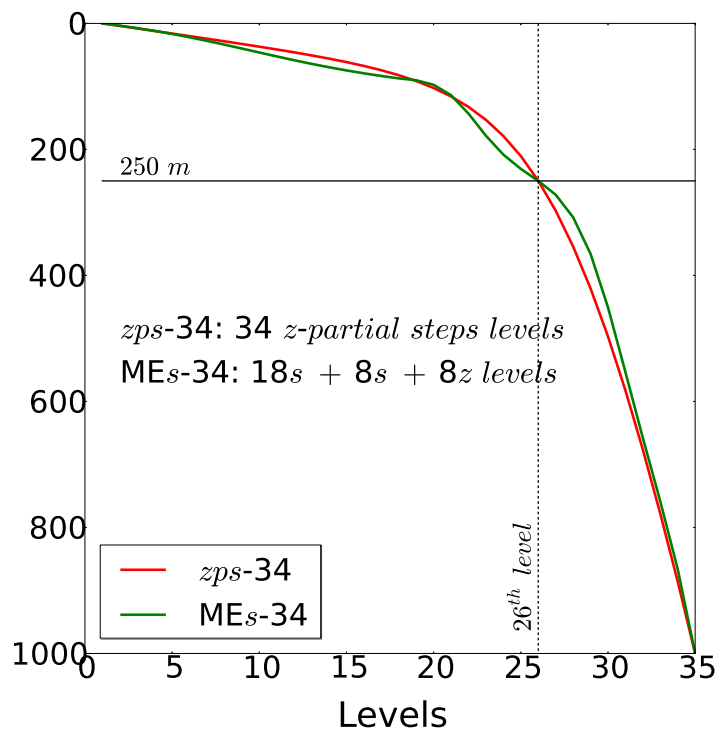

Fig. 5 Vertical distribution of zps-34 (red) and MEs-34 (green) model levels in the middle of the computational domain. The depth of the $26^{t h}$ level $(250 \mathrm{~m})$ is also shown.

One experiment is also carried out with a $z$-full step vertical grid at a very high vertical resolution of 150 levels (hereafter $z f s-150$ ). This simulation is used as a reference where analytical solutions are not available (see Section 3.2).

The $z p s-34$ grid uses a standard NEMO-3.6 z-partial steps scheme (Figure $4(\mathrm{c}))$ with a minimum layer thickness of $4 \mathrm{~m}$. The partial step parameters are tuned in such a way that the topography represented with 34 levels is close to the one discretised with 150 geopotential levels.

The MEs-34 grid is configured by using three envelopes (see Figure 4(d)). The middle $H_{e}^{2}$ and the deep $H_{e}^{3}$ envelopes are horizontal and located at $250 \mathrm{~m}$ and $1000 \mathrm{~m}$ respectively. Therefore, the deeper $D_{3}$ zone of the MEs grid is effectively discretised with a $z$-coordinate grid. The upper envelope $H_{e}^{1}$ of the MEs-34 grid is dome-shaped in the ocean interior, following a typical shape of the thermocline in a sea with a cyclonic circulation, but it follows an 'enveloping'-bathymetry over the continental slope and shelf.

The 'enveloping'-bathymetry is a smoothed version of the actual bathymetry with a maximum depth of $200 \mathrm{~m}$ and a minimum depth of $10 \mathrm{~m}$. It is obtained by applying the Martinho and Batteen (2006) smoothing algorithm to the actual topography, which reduces the maximum value of the slope parameter (Mellor et al., 1998) defined as

$$
r \equiv \frac{\left|H_{b}-H_{a}\right|}{H_{b}+H_{a}}
$$

where $H_{a}$ and $H_{b}$ are the depths of adjacent grid cells. With the $H_{e}^{1}$ envelope, the value of $r$ is reduced from $r=0.13$ (actual bathymetry) to 0.09 ( $H_{e}^{1}$ envelope), allowing the reduction of pressure gradients errors. 


\begin{tabular}{|c|c|c|c|c|c|}
\hline $\begin{array}{c}\text { Exp. } \\
\text { Name }\end{array}$ & $\begin{array}{l}\text { Oceanic } \\
\text { process }\end{array}$ & $\begin{array}{c}\text { Ideal test } \\
\text { process }\end{array}$ & $\begin{array}{c}\text { Initial ocean } \\
\text { setup }\end{array}$ & Perturb. & $\begin{array}{c}\text { Assess. of } \\
\text { models'skills }\end{array}$ \\
\hline $\begin{array}{c}\text { HPGE } \\
\text { (Sec. 3.2.1) }\end{array}$ & $\begin{array}{l}\text { Ocean } \\
\text { circulat. }\end{array}$ & $\begin{array}{l}\text { Evolution } \\
\text { of a stably } \\
\text { stratified } \\
\text { ocean } \\
\text { at rest }\end{array}$ & $\begin{array}{l}\text { Horiz. uniform } \\
\text { vert. stable } \\
\text { stratification, } \\
\text { no motion }\end{array}$ & - & $\begin{array}{l}\text { Comparison } \\
\text { with } \\
\text { analytical } \\
\text { solution }\end{array}$ \\
\hline $\begin{array}{c}\text { CASC } \\
\text { (Sec. } 3.2 .2)\end{array}$ & $\begin{array}{l}\text { Dense } \\
\text { water } \\
\text { cascading } \\
\text { upon the } \\
\text { shelf }\end{array}$ & $\begin{array}{l}\text { Gravity } \\
\text { current } \\
\text { over } \\
\text { steep } \\
\text { topography }\end{array}$ & $\begin{array}{l}\text { No stratific., } \\
\text { no motion }\end{array}$ & $\begin{array}{l}\text { Dense ring } \\
\text { upon the } \\
\text { shelf and the } \\
\text { shelf-break }\end{array}$ & $\begin{array}{l}\text { Comparison } \\
\text { with } \\
\text { analytical } \\
\text { solution }\end{array}$ \\
\hline $\begin{array}{c}\text { CILF } \\
\text { (Sec. 3.2.3) }\end{array}$ & $\begin{array}{l}\text { CIL } \\
\text { formation } \\
\text { in the } \\
\text { ocean } \\
\text { interior }\end{array}$ & $\begin{array}{l}\text { Sinking and } \\
\text { spreading of } \\
\text { a dense } \\
\text { cold patch }\end{array}$ & $\begin{array}{l}\text { Cyclonic } \\
\text { ocean with } \\
2 \text { density } \\
\text { layers }\end{array}$ & $\begin{array}{l}\text { Cylindrical } \\
\text { dense water } \\
\text { patch in the } \\
\text { upper layer }\end{array}$ & $\begin{array}{l}\text { Comparison } \\
\text { with high } \\
\text { vert. resol. } \\
\text { model solut. }\end{array}$ \\
\hline
\end{tabular}

Table 1 Oceanic processes tested in this study together with the associated experiment setup and the method used to evaluate models skills.

The uppermost envelope $H_{e}^{1}$ has a parabolic shape in deep areas $\left(H_{B}(x, y) \geq\right.$ 200) given by equation

$$
H_{e}^{1}=A+B\left(x^{2}+y^{2}\right)
$$

where $A=87.22$ and $B=273.33$. The MEs-34 configuration uses 18 levels in the upper $\left(D_{1}\right)$ zone, 8 levels in the central $\left(D_{2}\right)$ zone and 8 levels in the deeper $\left(D_{3}\right)$ zone. The configurations of the two 34 levels vertical grids are presented in Figure 5 , where the vertical distributions of zps-34 (red) and MEs-34 (green) model levels in the middle of the computational domain are compared.

The $z f s-150$ model uses a standard NEMO-3.6 $z$-full step grid (Madec, 2008) with the stretched function tuned in such a way that layers thickness up to $200 \mathrm{~m}$ depth is almost constant with a value of $\approx 2 \mathrm{~m}$ (Figure $4(\mathrm{~b})$ ).

\subsection{Experiment set-up}

We carry out three idealised process-oriented numerical experiments which mimic three typical oceanic conditions. The first experiment (hereinafter called HPGE) is designed to assess the generation of spurious currents due to horizontal pressure gradient errors (see Section 3.2.1). The second experiment (hereafter called CASC) represents dense water cascading from the continental shelf (Ivanov et al., 2004), see Section 3.2.2. The third experiment (hereinafter CILF) simulates the formation of a cold intermediate layer over a permanent thermocline, a process observed in many subarctic seas (Chubarenko and Demchenko, 2010; Cyr et al., 2011). The latter process is monitored in our simulations by using a passive tracer (see Section 3.2.3). The inventory of the experiments is given in Table 1.

The skills of MEs-34 and zps-34 models are assessed by comparison with known analytical solutions for the first and the second experiments. In the third experiment the analytical solution is not available and the comparison is made against a reference numerical simulation which uses $z f s-150$.

In all the numerical experiments, the time-splitting formulation for the nonlinear free surface is applied, with the baroclinic and barotropic time-steps equal 


\begin{tabular}{llll}
\hline \hline $\begin{array}{l}\text { Physical and Comput. } \\
\text { NEMO specific setup }\end{array}$ & HPGE EXP. & CASC EXP. & CILF EXP. \\
\hline \hline EOS & non-linear & non-linear & linear \\
& (TEOS10) & (TEOS10) & $\begin{array}{l}\text { Roquet et al., 2015) } \\
\lambda_{1}=\lambda_{2}=0.0 \\
\mu_{1}=\mu_{2}=\nu=0.0\end{array}$ \\
& & & $10^{-7}\left[\mathrm{~m}^{2} \mathrm{~s}^{-1}\right]$ \\
\hline Lateral diffusivity & $8\left[\mathrm{~m}^{2} \mathrm{~s}^{-1}\right]$ & $8\left[\mathrm{~m}^{2} \mathrm{~s}^{-1}\right]$ & $10^{-7}\left[\mathrm{~m}^{2} \mathrm{~s}^{-1}\right]$ \\
\hline Vertical diffusivity & $10^{-7}\left[\mathrm{~m}^{2} \mathrm{~s}^{-1}\right]$ & $\mathrm{GLS}$ & $10^{-5}\left[\mathrm{~m}^{2} \mathrm{~s}^{-1}\right]$ \\
\hline Vertical viscosity & $10^{-5}\left[\mathrm{~m}^{2} \mathrm{~s}^{-1}\right]$ & GLS & $10^{-1}$ \\
\hline
\end{tabular}

Table 2 Physical and computational NEMO setup specific of the three experiments. If not specified, NEMO standard values are used (see Madec 2008).

to $150 \mathrm{~s}$ and $7.5 \mathrm{~s}$, respectively. The Asselin time filter parameter is 0.1 . We use the pressure Jacobian scheme together with a leapfrog time scheme for calculation of the hydrostatic pressure gradient term. The Total Variance Dissipation (TVD) and Energy and ENstrophy (EEN) conservative schemes are used for tracer and momentum advection, respectively. All the simulations are performed using the $f$-plane approximation $\left(f \approx 10^{-4}\right)$. For the lateral diffusion of momentum, we use a second order operator aligned with horizontal levels together with a forth order operator discretised along model levels (O'Dea et al., 2012). The Laplacian and bi-laplacian viscosity coefficients are constant with values equal to $10^{2}\left[\mathrm{~m}^{2} \mathrm{~s}^{-1}\right]$ and $-2 \cdot 10^{9}\left[\mathrm{~m}^{4} \mathrm{~s}^{-1}\right]$, respectively. The lateral diffusion is simulated by using a horizontal harmonic operator with constant diffusivity (see Table 2 for the values used in each experiment). The vertical diffusivity and viscosity coefficients are constant in the HPGE and CILF experiments while are computed using the Generic Length Scale (GLS) turbulent closure scheme (Umlauf and Burchard, 2003, 2005) tuned following Wobus et al. (2013) in the CASC experiment (see Table 2). In the HPGE and CILF experiments we reduce the explicit vertical diffusivity to the minimum value allowed by model stability $\left(10^{-7}\left[\mathrm{~m}^{2} \mathrm{~s}^{-1}\right]\right)$, in order to isolate the effect of spurious numerical diffusion linked to the vertical discretisation scheme. All the models use no-slip lateral boundary conditions and a log-layer enhanced quadratic bottom friction parametrisation with minimum and maximum bottom drag coefficient values equal to $2.5 \cdot 10^{-3}$ and $10^{-1}$, respectively. Convection is parameterised by applying enhanced vertical diffusion on tracers in regions where the stratification is unstable. The enhanced vertical mixing coefficient is set equal to $10 \mathrm{~m}^{2} \mathrm{~s}^{-1}$.

\subsubsection{Generation of spurious currents}

In this experiments we assess the accuracy of the zps and MEs vertical schemes in representing horizontal pressure gradients. In zps models, the near bottom grid points within a vertical level are not necessarily at the same depth as the grid points in the interior, resulting in problems with pressure gradient errors and spurious diapycnal diffusion (Pacanowski et al., 1998).

The initial condition for each run is obtained by horizontally spreading the temperature and salinity profiles showed in Figure 6, so that there are no horizontal pressure gradients, there is no initial circulation and the sea surface is flat. There is no meteorological forcing or river discharge. In the absence of any external forcing, the analytical solution for current velocities and horizontal density gradients is zero. However, numerical errors due to the vertical discretisation may lead to 
errors in the pressure gradient computation, generating spurious current velocities (see for example Berntsen 2002).

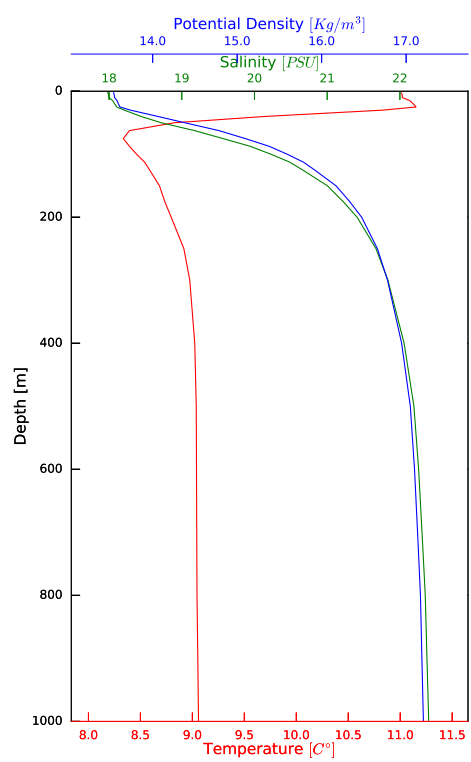

Fig. 6 Vertical profiles of temperature, salinity and potential density anomaly used as initial condition for the HPGR experiment. They are basin averaged mean annual climatologies computed from MyOcean Black Sea Reanalysis from 1992 to 2012 (MyOcean2, 2014).

The HPGE experiments consist of two prognostic simulations, one for each vertical grid, where the NEMO model is run for 30 days without any external forcing. The computational and physical NEMO settings are listed in Table 2 (HPGE experiments).

\subsubsection{Dense water cascading on the shelf}

In the second experiment we investigate the ability of the two 34 levels models to properly represent the flow of dense water down a steep topographic slope.

We consider an initial axisymmetric, three-dimensional density ring of dense water with a homogeneous density $\rho+\Delta \rho$, situated upon the shelf and an ambient ocean with constant density $\rho$. The initial velocity is zero everywhere.

The initial condition used for the numerical simulations is shown in Figure 7. The axisymmetrical dense ring is confined in coastal areas, has a maximum depth of $50 \mathrm{~m}$ and temperature, salinity and potential density anomaly $\sigma_{r}$ of $10^{\circ} \mathrm{C}, 21$ $P S U$ and $16.00 \mathrm{~kg} \mathrm{~m}^{-3}$, respectively. Ambient water temperature is $12^{\circ} \mathrm{C}$ and salinity is $20 \mathrm{PSU}$, yielding a potential density anomaly of $\sigma_{o}=14.94 \mathrm{~kg} \mathrm{~m}^{-3}$.

If such initial condition is allowed to evolve freely, the dense water will tend to descend downslope driven by the gravitational force while the Coriolis force will deflect such motion toward the right (Northern hemisphere). In the absence 


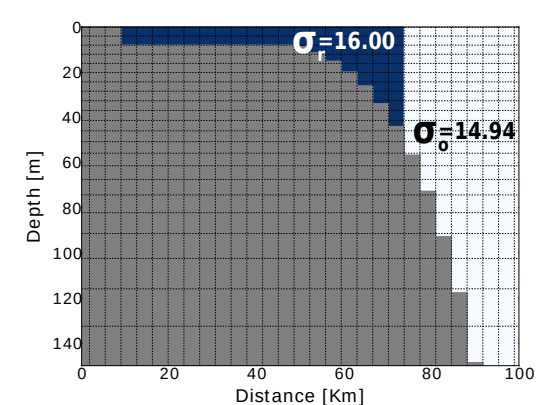

(a)

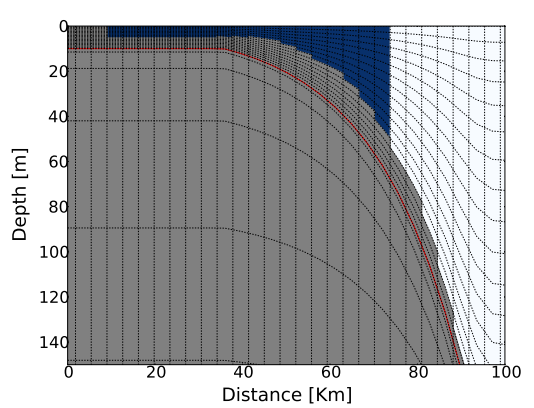

(b)

Fig. 7 Meridional cross-sections in the middle of the domain of the potential density initial condition for CASC experiments.

of friction an equilibrium eventually will be reached. For a constant bottom slope angle $\theta$, the geostrophic current velocity $u_{g}$ is given by $u_{g}=\frac{g^{\prime}}{f} \tan \theta$ (Nof, 1983), where $g^{\prime}$ is the reduced gravity $g^{\prime}=\frac{g \Delta \rho}{\rho_{0}}$ and $f$ is the Coriolis parameter. In the presence of friction, a tongue of dense water of approximately 2 Ekman depths will continue to descend.

In the case of a fully developed cascading without entrainment and ambient current, there is an analytical solution for the downslope velocity (Shapiro and Hill, 1997) given by

$$
u_{S H 97}=0.2 u_{g}
$$

where $u_{g}$ is the along slope geostrophic velocity (Nof, 1983). While the formula by Shapiro and Hill (1997) was derived for constant slopes, we compare our model results against this formula as the horizontal curvature of our domain is negligible as compared to the topographic slope, and the change of the slope over the length of the tongue is small. A similar approach was used in Wobus et al. (2011, 2013).

One month-long NEMO runs are performed with the computational and physical settings listed in Table 2 (CASC experiments). The GLS turbulence closure scheme is configured according to Wobus et al. (2013). The convective adjustment parameterization is used following Laanaia et al. (2010). The experiment is conducted with 2 vertical grids, the $z p s-34$ and the MEs-34.

\subsubsection{Formation of Cold Intermediate Layer}

In the third experiment, we assess the ability of the $z p s-34$ and MEs-34 vertical grids to represent the formation of a Cold Intermediate Layer (CIL) over a permanent pycnocline by monitoring the advection of a passive tracer in the ocean interior. The experiment simulates the sinking and spreading of a dense (cold) patch of water in an idealised cyclonic ocean with a doming pycnocline.

The initial condition is axisymmetric and represents a two-layer fluid with a cold cylindrical patch at the centre of the basin (see Figure 8 ). The main pycnocline is defined by Equation 9 with $A=92.92$ and $B=193.33$ (note: the pycnocline does not coincide with the $H_{e}^{1}$ envelope). Salinity is equal to $35 \mathrm{PSU}$ and is constant everywhere. The initial velocity is zero. We use a linear equation of state with 


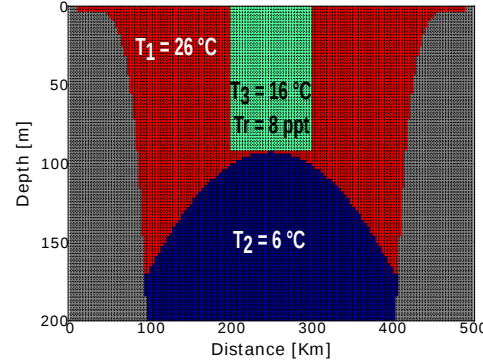

(a)

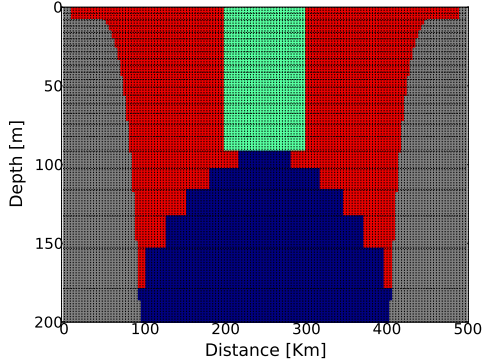

(b)

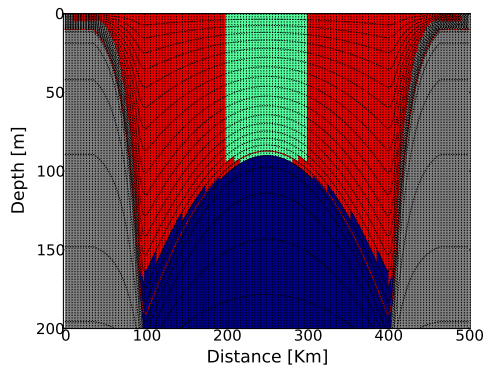

(c)

Fig. 8 Meridional cross-sections in the middle of the domain of temperature and salinity initial condition fields defined on $z f s-150$ (a), zps-34 (b) and MEs-34 (c) models' grids for the CILF experiment.

coefficients shown in Tab. 2 (CILF experiment). Temperature and density anomaly above (i.e. in layer 1 ) and below (layer 2) the pycnocline are $T_{1}=26^{\circ} \mathrm{C}, \sigma_{1}=$ $23.4 \mathrm{~kg} \mathrm{~m} \mathrm{~m}^{-3}$ and $T_{2}=6^{\circ} \mathrm{C}, \sigma_{2}=26.7 \mathrm{~kg} \mathrm{~m}^{-3}$, respectively. The cylindrical dense convective patch has a radius of $50 \mathrm{~km}$, a maximum depth of $92.94 \mathrm{~m}$ and temperature, density anomaly and passive tracer concentration equal to $T_{3}=$ $16^{\circ} \mathrm{C}, \sigma_{3}=25.0 \mathrm{~kg} \mathrm{~m} \mathrm{~m}^{-3}$ and $C=8 \mathrm{ppt}$, respectively. The ratio between the volume of the cold dense patch (green slug in Figure 8) and the volume of the domed denser layer (blue fluid portion in Figure 8) is 0.011 in all the models.

Explicit tracer diffusion is negligibly small in order to isolate the numerical diffusion linked to advection schemes. However, we use a standard high value (10 $m^{2} s^{-1}$ ) of vertical diffusivity for convective adjustment. The computational and physical settings are listed in Table 2 (CILF experiment). We use the numerical solution of the very high vertical resolution $z f s-150$ model as a reference to evaluate the performance of both $z p s-34$ and MEs-34 vertical schemes.

The numerical simulations are performed for 60 days. When the lateral exchange and spreading of an oceanic cold water patch occurs, baroclinic instabilities break up the mixed patch and homogeneous water sinks and spreads out at its neutrally buoyant level (see fig. 3 in Marshall and Schott 1999). 


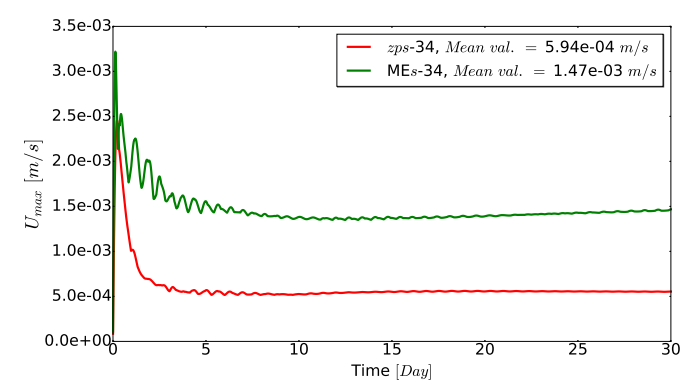

(a)

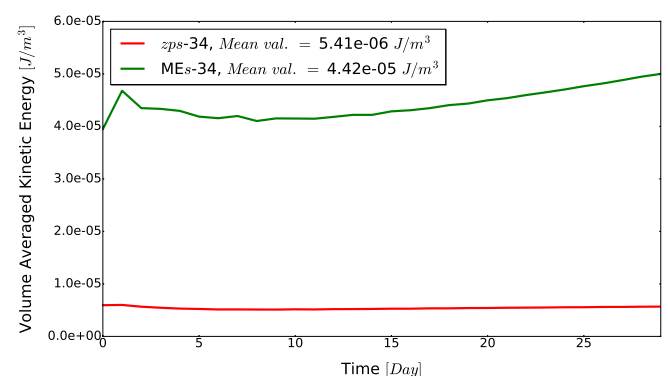

(b)

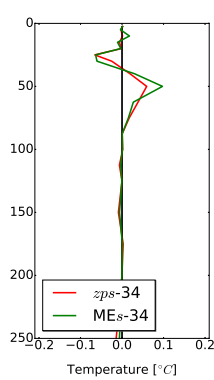

(c)

Fig. 9 (a) Time series of spurious currents maximum values, (b) time series of basin averaged Kinetic Energy and (c) differences between temperature profiles extracted in the middle of the domain after 30 days of simulation and the initial condition of $z p s-34$ (red) and MEs-34 (green) models.

\section{Results and Discussion}

\subsection{Horizontal pressure gradients errors}

The numerical results of this experiment demonstrate that horizontal pressure gradient errors appear in both MEs and zps models. After 31 days, spurious currents develop in both models, however their absolute values are small in both cases. In the $z p s$-34 model they are localized only in proximity of the sloping sea-floor while in the MEs-34 model they affect all the domain.

Time series of the maximum value of spurious currents computed over the whole domain (Figure 9(a)) show that $u_{\max }$ values are less than $5 \cdot 10^{-3} \mathrm{~ms}^{-1}$, i.e. well within the acceptable margin of error and are comparable with the accuracy of high-precision instruments (Valeport, 2017). The averaged over the length of the simulation maximum error for the zps-34 model is $0.59 \cdot 10^{-3} \mathrm{~ms}^{-1}$, which is slightly better than the one of the MEs model, where the average maximum value is $1.47 \cdot 10^{-3} \mathrm{~ms}^{-1}$.

The time series of the basin averaged Kinetic Energy (KE) due to spurious currents are compared in Figure 9(b). The zps-34 model has a time averaged KE of $5.41 \cdot 10^{-6} \mathrm{Jm}^{-3}$, which corresponds to an average speed of $1.02 \cdot 10^{-4} \mathrm{~ms}^{-1}$. The MEs-34 model shows slightly higher but still very low values: basin averaged $\mathrm{KE}$ of $4.42 \cdot 10^{-5} \mathrm{Jm}^{-3}$ and average speed of $2.93 \cdot 10^{-4} \mathrm{~ms}^{-1}$. After one month of simulation, the KE in the MEs model does not reach an equilibrium. In the case of $\sigma$-coordinates, this behaviour has been classified as sigma error of the second kind (SESK) (Mellor et al., 1998) and it has been reported and studied in a number of publication (see for example Shchepetkin 2003 and references therein).

Figure $9(\mathrm{c})$ presents differences between the temperature profiles extracted in the middle of the domain of the two models after 30 days of simulation and the initial condition, showing that the same level of spurious mixing is obtained with both the models.

As discussed in Sec. 3.1, the doming of the computational levels in MEs-34 was introduced to deal with ocean domains characterized by a cyclonic circulation. In 


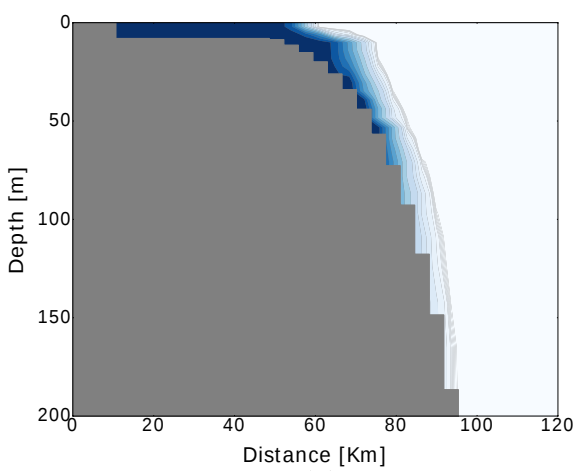

(a)

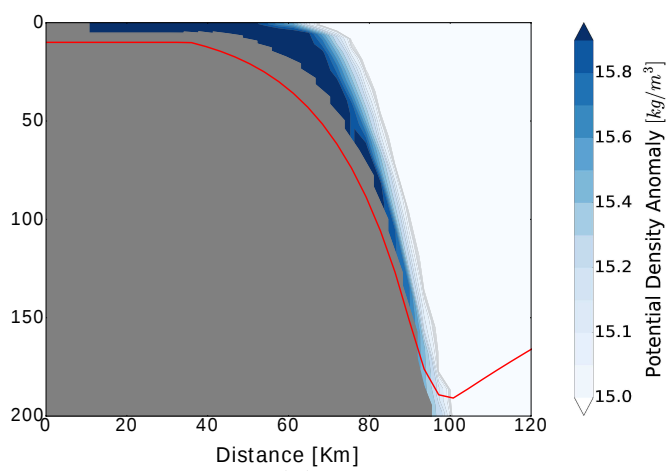

(b)

Fig. 10 Cross section in the middle of the domain showing the cascade simulated by the zps-34 (a) and the MEs-34 (b) models at day 6.

this experiment we use MEs-34 for an ocean with largely horizontal isopycnals and an absence of any background circulation. In order to evaluate a potential negative effect of curved computational levels in the ocean interior we also performed an additional simulation with the same grid set-up of the MEs-34 grid but using a modified upper envelope $H_{e}^{1}$ which is horizontal in the ocean interior. Hereinafter we call this grid SH13-34, since it follows Shapiro et al. (2013), see Figure 1(d). Comparisons of numerical results obtained with the MEs-34 and the SH13-34 grids demonstrate that inclining the model levels in the ocean interior (used in MEs-34) does not increase the magnitude of spurious currents. The time-averaged maximum value of spurious currents in the $\mathrm{SH} 13-34$ is $1.46 \cdot 10^{-3} \mathrm{~ms}^{-1}$ as compared to $1.47 \cdot 10^{-3} \mathrm{~ms}^{-1}$ in MEs-34. This result supports the use of MEs-34 type models with the curved upper envelope even in areas without cyclonic circulations or where ocean fronts are weak or moderate.

\subsection{Dense water cascading on the shelf}

We evaluate the zps-34 and MEs-34 models' performance in representing dense water overflows down a steep topography by comparing the numerical results of the downslope velocity with theoretical values given by Shapiro and Hill (1997).

The downslope speed is defined as the speed of the plume head in an azimuthally averaged sense. The plume is defined as a water mass with potential density $\geq 1014.99 \mathrm{~kg} \mathrm{~m}^{-3}$. The speed is computed using the horizontal distance of each grid cell representing the plume head from the middle of the domain.

Time series of the plume edge depths show that both models reproduce a dense water cascading with nearly constant downward speed (Figure 10). The plume head reaches the deepest zone of the model topography $(1000 \mathrm{~m})$ after 11 days in the case of the MEs-34 model and after 14 days with the zps-34 grid.

In order to compare the numerical and analytical solutions, we compute the downslope velocity $u_{\text {model }}$ of the simulated cascades only when the plume edge is located in areas where the topographic slope is between 0.006 and 0.020 and the depth is less then $800 \mathrm{~m}$ (see Figure 11(a)). To compute the Nof's velocity we use a slope of 0.014 , the mean value of the actual bottom slope. 


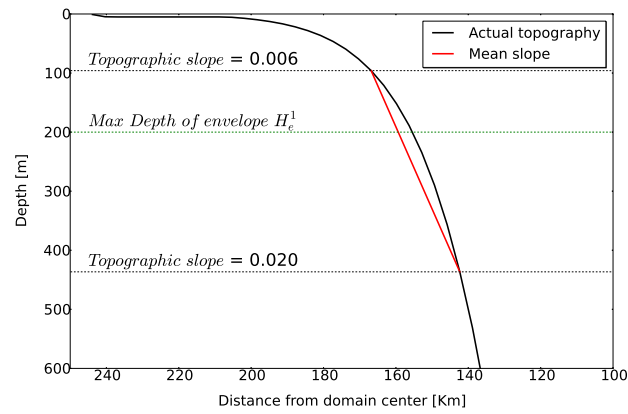

(a)

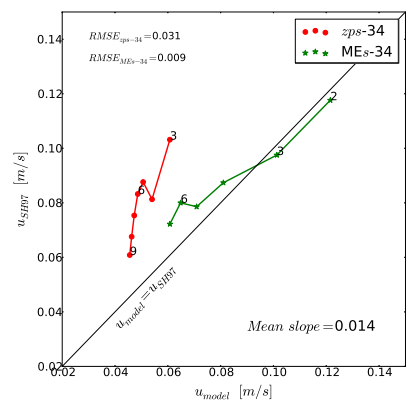

(b)

Fig. 11 (a) Actual topography of the model domain (black) and the slope used to compute the Nof (1983) velocity (red). The locations where the topographic slope is equal to 0.006 and 0.020 and the maximum depth of envelope $H_{e}^{1}$ of the MEs model are shown as well. (b) Comparison between the daily values of the downslope velocity predicted by the Shapiro and Hill (1997) theoretical model with the numerical ones obtained with the $z p s-34$ (red) and the MEs-34 (green) models. Numbers indicate the day.

In order to compute the reduced gravity $g^{\prime}$, we consider a reference potential density $\rho_{0}$ given by the daily mean of azimuthally-averaged potential densities in model cells just above the model bathymetry. The ambient water density $\rho_{a}$ is obtained by computing the daily mean of azimuthally-averaged potential densities in model bottom cells with values less than $1014.99 \mathrm{~kg} \mathrm{~m}^{-3}$. Finally, the daily potential density $\rho_{c}$ representative of the dense cascade of each model run is computed by daily averaging potential densities of bottom cells where the azimuthally-averaged potential density is between 1015.35 and $1014.99 \mathrm{~kg} \mathrm{~m}^{-3}$.

Figure 11(b) shows the comparison between the daily values of the downslope velocity given by the analytical solution (Shapiro and Hill, 1997) and the numerical solutions obtained with the zps-34 and the MEs-34 models.

Results show that the MEs-34 model performs significantly better than the zps-34 model. In the zps-34 model, the dense water cascade crosses the analysed zone (i.e. the area between the water depths of 90 and $450 \mathrm{~m}$, see Figure11(a)) from day three to day 9. Throughout the entire period, the $z p s-34$ underestimates the downslope speed of cascading, especially in the beginning of the event (day 3). The RMS error of the $z p s-34$ model is $0.031 \mathrm{~ms}^{-1}$, which is high (about $50 \%$ ) compared to the average downslope speed of $0.05-0.07 \mathrm{~ms}^{-1}$. On the other hand, in the MEs-34 model the plume descends faster, has lower loss of density due to entrainment, and crosses the analysed zone from day 2 to 7 . The modelled downslope speeds are in the range of $0.06-0.12 \mathrm{~ms}^{-1}$ and are almost equal to the analytical solution, with a RMS error of $0.009 \mathrm{~ms}^{-1}$, or about $10 \%$ of the average speed. The fact that the downslope cascading in zps-34 is slower than in MEs-34 is probably due to the enhanced artificial mixing (reducing $g^{\prime}$ ) which characterises $z$-type models with step-like topography (see Figure 10). This agrees with other gravity current overflow experiments results (see for example fig. 2 in Ezer 2005).

Figure 11(b) shows that during the days 6 and 7 of the MEs-34 simulation, the plume reaches the lower computational zone D2, which has some horizontal (geopotential) levels. The accuracy of the simulation slightly decreases at this point 


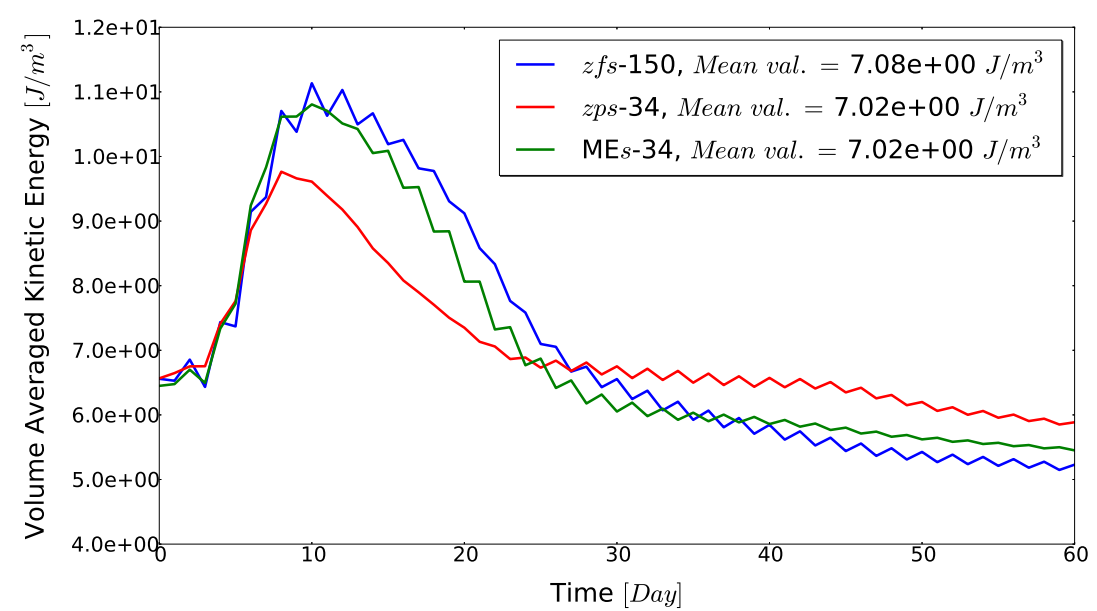

Fig. 12 Time series of the volume averaged KE for the $z f s-150$ (blue), zps-34 (red) and MEs-34 (green) models.

in time as the cascade head reaches a point in the vertical coordinate system which begins to resemble a $z$-level grid.

\subsection{Formation of Cold Intermediate Layer}

For this experiment, an analytical solution is not available. Therefore, we compare the results of $z p s-34$ and MEs-34 models with the reference solution produced by the high resolution $z f s-150$ model.

A $z f s-150$ simulation is significantly more expensive computationally than a simulation performed with the other two low resolution models. In this experiment for example, the duration of the $z f s$ - 150 simulation on our HPC cluster was 70556 $s(\approx 19.6 \mathrm{hr})$, while $z p s-34$ and MEs-34 numerical runs took $17579 s(\approx 4.9 \mathrm{hr})$ and $21646 s(\approx 6.0 \mathrm{hr})$, respectively.

We begin the analysis with the comparison of the 60 days long time series of the volume averaged Kinetic Energy (KE) of the three models (Figure 12).

After a few days of spin-up, all the simulations seem to represent the same general dynamics: a first energetic stage where the dense cold patch sinks and spreads along the permanent pycnocline and a second less active regime where the CIL is at its neutrally buoyant level and geostrophy is the leading dynamics.

The time series of basin averaged KE produced with MEs-34 and the reference $z f s-150$ models are quite similar, with a RMS error equal to $0.15 \mathrm{Jm}^{-3}$ (or approximately $2 \%$ of the mean $\mathrm{KE}$ ). Both models show a maximum of $\mathrm{KE}$ at day 10 with values of $10.81 \mathrm{Jm}^{-3}$ in the case of the MEs-34 model and $11.13 \mathrm{Jm}^{-3}$ for the reference $z f s-150$ model.

On the other hand, the $z p s-34$ model simulates a shorter and less energetic first phase and a moderately more vigorous geostrophic stage, with a RMS error of 0.96 $\mathrm{Jm}^{-3}$ (or $14 \%$ of the mean KE). The maximum of KE in the $z p s-34$ simulation is $9.76 \mathrm{Jm}^{-3}$ and is reached at day 8, i.e. 2 days earlier than the reference. 


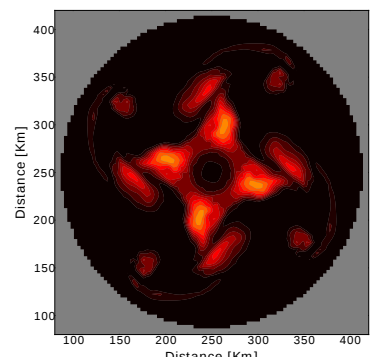

(a)

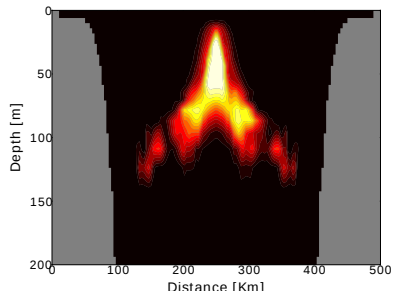

(d)

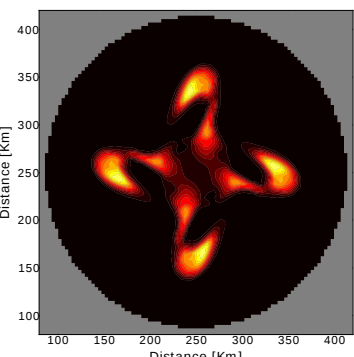

(b)

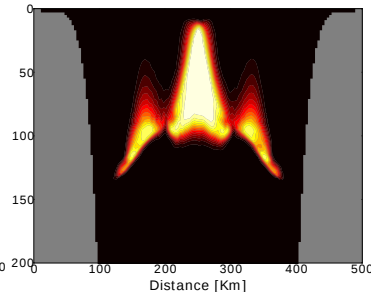

(e)

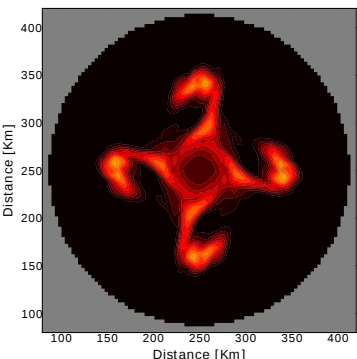

(c)

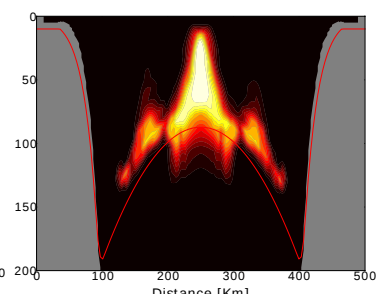

(f)

Fig. 13 Passive tracer concentration after 18 days. First row: horizontal distribution maps obtained at $105 \mathrm{~m}$ depth with the zps-34 (a), the $z f s-150$ (b) and the MEs-34 (c) models. Second row: meridional cross sections obtained with the $z p s-34(\mathrm{~d})$, the $z f s-150(\mathrm{e})$ and the MEs-34 (f) models.

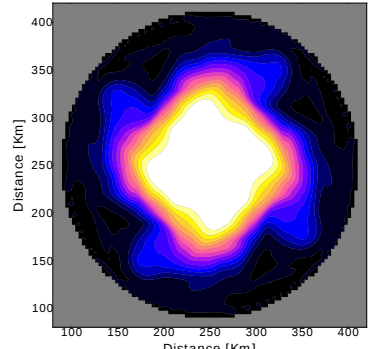

(a)

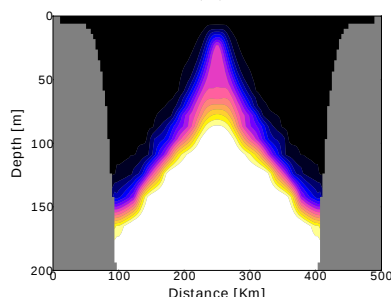

(d)

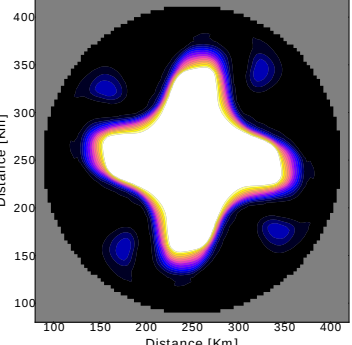

(b)

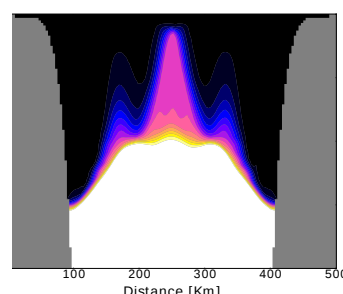

(e)

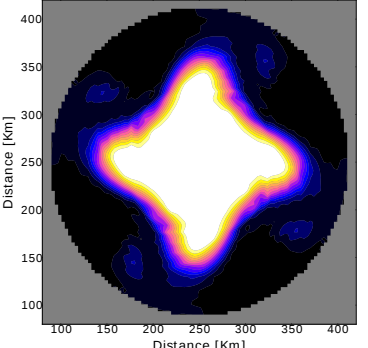

(c)

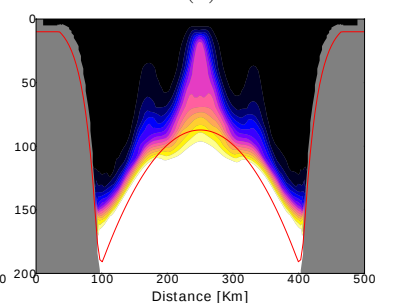

(f)

Fig. 14 Density anomaly distribution after 18 days. First row: horizontal maps at a depth of $120 \mathrm{~m}$ obtained with the zps-34 (a), the $z f s-150$ (b) and the MEs-34 (c) models. Second row: meridional cross sections obtained with the $z p s-34$ (d), the $z f s-150$ (e) and the MEs-34 (f) models. 
Daily averaged horizontal distribution maps and vertical cross sections of density anomaly and passive tracer concentration after 18 and 50 days illustrate how the more energetic (day 18) and the less dynamical (day 50) stages of the CIL formation are represented by the three models.

After 18 days, the $z f s-150$ and MEs-34 models represent similar mesoscale baroclinic structures (see Figure 13(b)-(c) and Figure 14(b)-(e)-(d)-(f)). As expected, the high resolution reference model $z f s-150$ is able to maintain the sharp pycnocline, both in the lateral and in the vertical directions (Figure 14(b)-(e)). The MEs-34 model demonstrates a similar capability, especially for horizontal gradients (Figure 14(c)-(f)). On the other hand, Figure 13(a) and Figure 14(a)-(d) show that the zps-34 model generates stronger diapycnal diffusion and entrainment than $\mathrm{MEs}-34$.

The transport of the passive tracer along the pycnocline after 18 days is similarly represented by both the $z f s-150$ and MEs-34 models (Figure 13(e)-(f)). To the contrary, the zps-34 model generates spurious mixed patches of tracer concentration shown in blue in Figure 13(d).

This effect is probably due to the fact that the horizontal computational levels create a staggered representation of the pycnocline, and hence are subject to the same spurious mixing as when $z$-levels hit the sloping bottom.

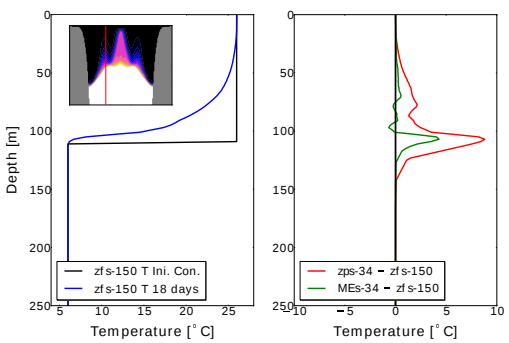

(a)

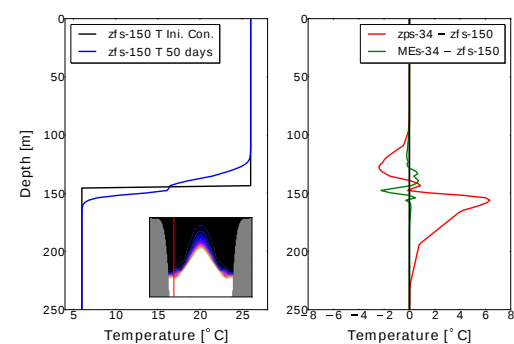

(b)

Fig. 15 Temperature vertical profiles simulated with the reference $z f s$-150 model (blue) and errors relative to the reference produced by the zps-34 (red) and the MEs-34 (green) models. The location of the profiles is shown in each cross section. (a) After 18 days and (b) after 50 days of simulations. The initial condition is shown in black.

Figure 15(a) shows a vertical profile of temperature simulated by the reference $z f s-150$ model in the proximity of the head of spreading dense water (blue profile, left sub-panel). It also shows the errors relative to the reference produced by the zps-34 (red) and MEs-34 (green) models, demonstrating that the MEs-34 grid has a significantly smaller error than the zps-34 model.

The vertical profiles of errors in horizontally averaged density and passive tracer concentration relative to the reference $z f s-150$ numerical solution after 18 days are presented in Figure 16(a)-(b). They show that the error generated by MEs-34 model is approximately $50 \%$ smaller in comparison to the zps-34 model.

At day 50, all three models simulate a less active dynamics, where the lateral exchange and spreading of the dense cold water to its neutrally buoyant level is 

18.

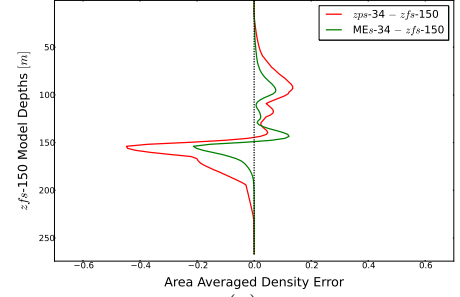

(a)

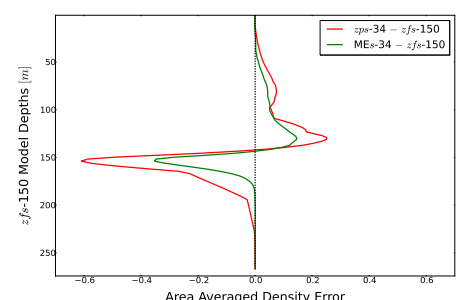

(c)

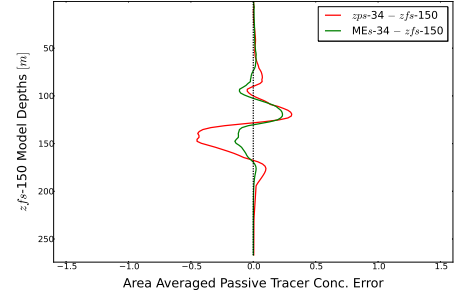

(b)

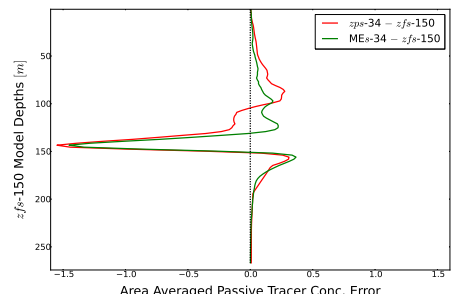

(d)

Fig. 16 Area averaged density and passive tracer concentration difference between the $z p s-34$ (red) and the MEs-34 solutions and the reference $z f s-150$ one after 18 days (a-b) and 50 days (c-d) of simulation.

The reference $z f s-150$ solution shows that after 50 days the initial dense cold patch has formed a nearly uniform well-defined density layer with sharp fronts above the main pycnocline (see Figure 18(b)-(e)). The passive tracer is advected with low numerical diffusion, reaching depths of around $150 \mathrm{~m}$ at almost the original concentration (Figure 17(b)-(e)).

Figures 17(a)-(d) show the impact of the higher numerical diffusion of the $z p s$ 34 model in the transport of the passive tracer: the nearly uniform distribution along the pycnocline of the reference solution is lost and the passive tracer is mostly confined at depths shallower than $120 \mathrm{~m}$. The maximum of tracer concentration is located at depths around $80-90 \mathrm{~m}$. Figure 16(d) confirms that this is the case for the whole domain: at day 50, the $z p s$-34 model simulates moderately higher tracer concentrations than the zfs-150 model at depths between 90-110 $\mathrm{m}$ and importantly lower values between 110-150 $\mathrm{m}$.

After 50 days, the MEs-34 model represents a nearly uniform tracer distribution along the main pycnocline up to 120-130 $m$ (see Figure 17(f)). The MEs-34 model simulates a horizontal passive tracer ring-shaped distribution at a depth of $120 \mathrm{~m}$ (Figure $17(\mathrm{c})$ ) which is very similar to the reference $z f s$-150 solution (Figure 17(b)). This proves a lower artificial diffusion of the MEs-34 model in comparison to the zps-34 one. Figure 16(d) shows that at day 50 both zps-34 and MEs-34 models generate slightly higher values than the reference solution. At depths around $150 \mathrm{~m}$, both zps-34 and MEs-34 simulate lower values than the reference. 


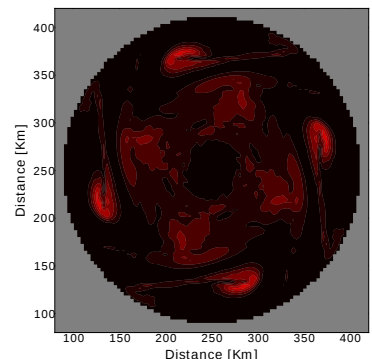

(a)

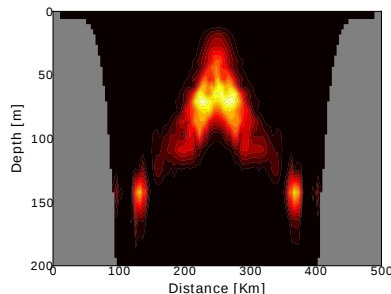

(d)

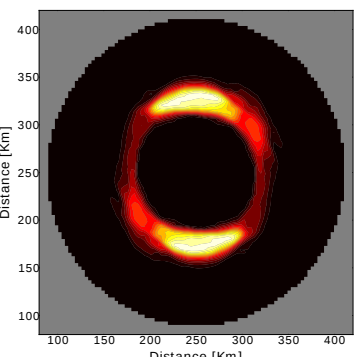

(b)

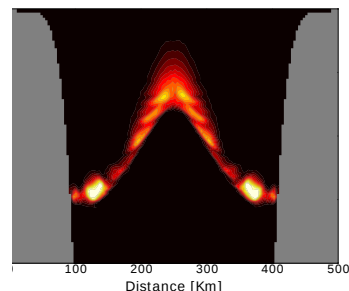

(e)

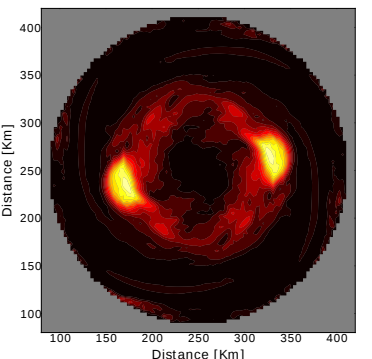

(c)

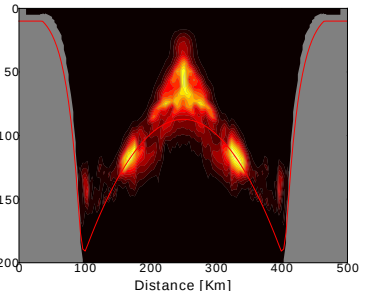

(f)

Fig. 17 Passive tracer concentration after 50 days. First row: horizontal distribution maps obtained at $120 \mathrm{~m}$ depth with the $z p s-34$ (a), the $z f s-150$ (b) and the MEs-34 (c) models. Second row: zonal cross sections obtained with the $z p s-34$ (d), the $z f s-150$ (e) and the MEs-34 (f) models.

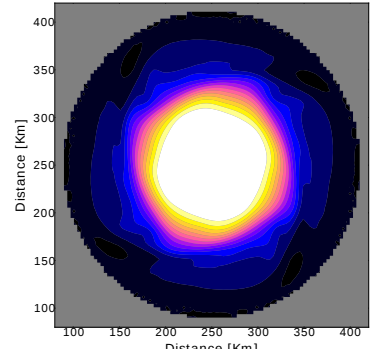

(a)

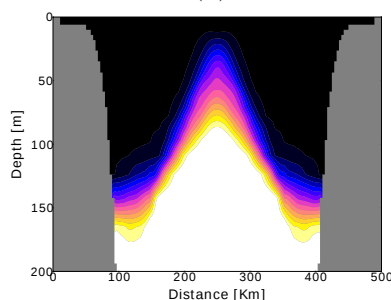

(d)

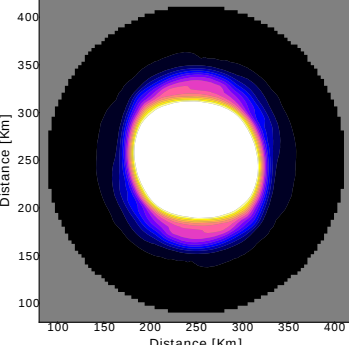

(b)

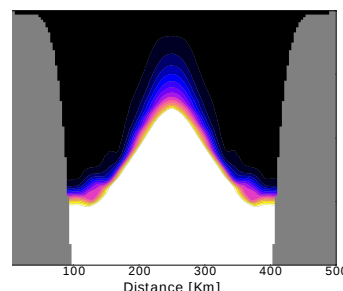

(e)

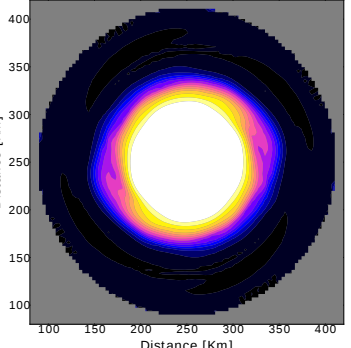

(c)

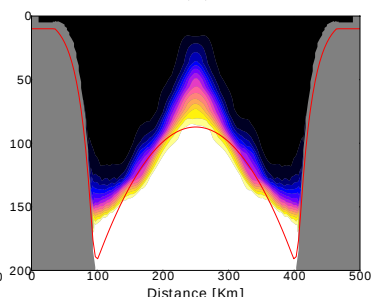

(f)

Fig. 18 Density anomaly distribution after 50 days. First row: horizontal maps at a depth of $120 \mathrm{~m}$ obtained with the zps-34 (a), the $z f s-150$ (b) and the MEs-34 (c) models. Second row: zonal cross sections obtained with the $z p s-34$ (d), the $z f s-150$ (e) and the MEs-34 (f) models. 
The zps-34 model shows the formation of a more extended and diffusive CIL, with weaker horizontal and vertical gradients (Figure 18(a)-(d)). These artefacts are the result of the low vertical resolution combined with the step-like representations of both pycnocline and advection.

A spurious downwelling event is produced with the zps-34 model, while it is not present either in the reference $z f s-150$ or MEs-34 solutions (see Figure 18(d)(e)-(f) and Figure 15(b)).

Figures 15(b), 16(c) and 18(c)-(f) show that the MEs-34 model simulates the formation of a CIL closer to the reference $z f s-150$ model, with lower diapycnal diffusion and sharper density fronts than the $z p s-34$ model.

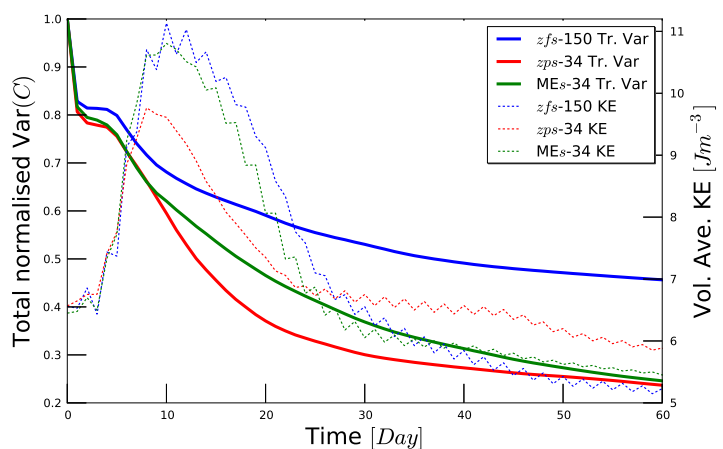

(a)

Fig. 19 Time series of the normalised passive tracer total variance (bold lines) and volume averaged KE (dashed lines) for the $z f s-150$ (blue), zps-34 (red) and MEs-34 (green) models. Normalisation is done with respect to the total variance of the initial condition.

The numerical mixing due to discretisation errors of tracer advection schemes causes decay in time of the passive tracer total variance (Maqueda and Holloway, 2006; Burchard and Rennau, 2008; Klingbeil et al., 2014), which is defined for a Boussinesq fluid as

$$
\operatorname{Var}(C)=\left\langle C^{2}\right\rangle_{V}-\langle C\rangle_{V}^{2}
$$

where $C$ is the concentration of the passive tracer and $\langle\bullet\rangle_{V}=V^{-1} \int \bullet d V$ represents a global averaging operator in a ocean with volume $V=\int d V$.

Following James (1996), in Figure 19 we compare the cumulative loss with time of the discrete passive tracer total variance in the three models in terms of the ratio $\operatorname{Var}(C)^{n} / \operatorname{Var}(C)^{0}$, where $n$ indicates the discrete time level and $n=0$ is the initial condition. Numerical results show that, as expected, the reference $z f s-150$ model has the lowest loss of variance with time, and hence the smallest numerical diffusion. The MEs-34 model performs generally better than zps-34, especially during the more dynamic phase of the simulation. Both models give similar results after the end of the active phase. 


\section{Conclusions}

In this study we present and assess the skills of a new vertical discretisation scheme which we call the 'Multi-Envelope $s$-coordinate system' or 'MEs'. Our new system further develops the earlier concept of 'enveloped bathymetry', where model levels followed a 'virtual bottom' (aka envelope) rather than the actual bathymetry. Such 'single-envelope' system could be classed as an extreme case of the new 'multienvelope' system. The multi-enveloping method allows the definition of computational surfaces which are optimised to best represent the physical processes in question. This method provides greater flexibility in the designing of a vertical grid than currently available geopotential level or terrain-following systems. All of these systems can be obtained as specific implementations of MEs.

An assessment of the MEs model skill for a number of idealized process studies shows that MEs generates a small pressure gradient error, gives a better representation of dense water cascades down the continental slope and provides a more accurate simulation of formation of a cold intermediate layer, than a comparable z-partial steps system.

The MEs systems allows achieving a quality of simulation similar to a standard geopotential grid which has a much higher number of levels, and hence the MEs system is more computationally efficient.

The algorithm of creating MEs was implemented in NEMO for this study, but can easily adapted for any 3D ocean model.

\section{Acknowledgements}

This work was funded by the EASME/EMFF/2014/1.3.1.3/LOT4/SI2.709436 Seabasin Checkpoints - Lot 4 - 'BLACK SEA' project. The authors are grateful to the valuable comments and suggestions of the two anonymous reviewers which have greatly contributed to improving the manuscript.

\section{Appendix 1}

For each $(x, y)$ of the horizontal domain the complete cubic spline $P_{x, y, i}^{3}\left(\sigma_{i}\right)$ of the vertical sub-zone $D_{i}$ can be written as

$$
P_{x, y, i}^{3}\left(\sigma_{i}\right)=a_{x, y, i}+b_{x, y, i}\left(-\sigma_{i}\right)+c_{x, y, i}\left(-\sigma_{i}\right)^{2}+d_{x, y, i}\left(-\sigma_{i}\right)^{3}
$$

where $\sigma_{i}$ is given by Equation 4 and $-1<\sigma_{i} \leq 0$.

Applying the three constraints defined in Section 2 leads to a tridiagonal linear system of four equations for the four unknowns $a_{x, y, i}, b_{x, y, i}, c_{x, y, i}$ and $d_{x, y, i}$ (de Boor, 1978).

A modified version of the Fortran90 numerical library PPPACK (de Boor, 1978) has been introduced in the NEMO code to compute the four coefficients of the complete cubic spline $P_{x, y, i}^{3}\left(\sigma_{i}\right)$. 


\section{References}

Adcroft, A. and Campin, J.-M. (2004). Rescaled height coordinates for accurate representation of free-surface flows in ocean circulation models. Ocean Modelling, 7(3-4):269-284.

Adcroft, A., Hill, C., and Marshall, J. (1997). Representation of Topography by Shaved Cells in a Height Coordinate Ocean Model. Monthly Weather Review, 125(9):2293-2315.

Barnier, B., Madec, G., Penduff, T., Molines, J.-M., Treguier, A.-M., Le Sommer, J., Beckmann, A., Biastoch, A., Böning, C., Dengg, J., Derval, C., Durand, E., Gulev, S., Remy, E., Talandier, C., Theetten, S., Maltrud, M., McClean, J., and De Cuevas, B. (2006). Impact of partial steps and momentum advection schemes in a global ocean circulation model at eddy-permitting resolution. Ocean Dynamics, 56(5-6):543-567.

Barnier, B., Marchesiello, P., De Miranda, A. P., Molines, J.-M., and Coulibaly, M. (1998). A sigma-coordinate primitive equation model for studying the circulation in the South Atlantic. Part I: Model configuration with error estimates. Deep Sea Research I, 45:543-572.

Berntsen, J. (2002). Internal pressure errors in sigma coordinate ocean models. Journal of Atmospheric and Oceanic Technology, 21(1994):1403-1413.

Bleck, R. (1998). Ocean modeling in isopycnic coordinates. In Chassignet, E. P. and Verron, J., editors, Ocean Modeling and Parameterization, NATO ASI Mathematical and Physical Sciences Series, Vol. 516, pages 423-448. Kluwer Academic Publishers, Dordrecht.

Bleck, R. (2002). An oceanic general circulation model framed in hybrid isopycnicCartesian coordinates. Ocean Modelling, 4(1):55-88.

Bleck, R. and Boudra, D. (1981). Initial Testing of a Numerical Ocean Circulation Model Using a Hybrid (Quasi-Isopycnic) Vertical Coordinate. Journal of Physical Oceanography, 11(6):755-770.

Blumberg, A. F. and Mellor, G. L. (1987). A description of a three-dimensional coastal ocean circulation model. In Three-Dimensional Coastal Ocean Models, pages $1-16$.

Burchard, H. and Rennau, H. (2008). Comparative quantification of physically and numerically induced mixing in ocean models. Ocean Modelling, 20(3):293-311.

Chassignet, E. P., Hurlburt, H. E., Smedstad, O. M., Halliwell, G. R., Hogan, P. J., Wallcraft, A. J., and Bleck, R. (2006). Ocean Prediction with the Hybrid Coordinate Ocean Model (HYCOM). In Chassignet, E. P. and Verron, J., editors, Ocean Weather Forecasting, pages 413-426. Springer-Verlag, Berlin/Heidelberg.

Chubarenko, I. and Demchenko, N. (2010). On contribution of horizontal and intra-layer convection to the formation of the Baltic Sea cold intermediate layer. Ocean Science, 6(1):285-299.

Cyr, F., Bourgault, D., and Galbraith, P. S. (2011). Interior versus boundary mixing of a cold intermediate layer. Journal of Geophysical Research, 116(C12):C12029.

de Boor, C. (1978). A Practical Guide to Splines. Springer-Verlag New York, New York.

Dukhovskoy, D. S., Morey, S. L., Martin, P. J., O'Brien, J. J., and Cooper, C. (2009). Application of a vanishing, quasi-sigma, vertical coordinate for simulation of high-speed, deep currents over the Sigsbee Escarpment in the Gulf of 
Mexico. Ocean Modelling, 28(4):250-265.

Enriquez, C. E., Shapiro, G. I., Souza, A. J., and Zatsepin, A. G. (2005). Hydrodynamic modelling of mesoscale eddies in the Black Sea. Ocean Dynamics, 55(5-6):476-489.

Ezer, T. (2005). Entrainment, diapycnal mixing and transport in three-dimensional bottom gravity current simulations using the Mellorâ $\breve{A S Y Y a m a d a ~ t u r b u l e n c e ~}$ scheme. Ocean Modelling, 9(2):151-168.

Ezer, T. (2016). Revisiting the problem of the Gulf Stream separation: on the representation of topography in ocean models with different types of vertical grids. Ocean Modelling, 104:15-27.

Ezer, T. and Mellor, G. L. (1992). A Numerical Study of the Variability and the Separation of the Gulf Stream, Induced by Surface Atmospheric Forcing and Lateral Boundary Flows. Journal of Physical Oceanography, 22(6):660-682.

Ezer, T. and Mellor, G. L. (2004). A generalized coordinate ocean model and a comparison of the bottom boundary layer dynamics in terrain-following and in z-level grids. Ocean Modelling, 6(3-4):379-403.

Furner, R. (2012). A review of lateral diffusion for Met Office ocean shelf modelling. Technical report, Met Office.

Gangopadhyay, A. and Robinson, A. R. (2002). Feature-oriented regional modeling of oceanic fronts. Dynamics of Atmospheres and Oceans, 36(1-3):201-232.

Gerdes, R. (1993a). A primitive equation ocean circulation model using a general vertical coordinate transformation: 1 . Description and testing of the model. Journal of Geophysical Research, 98(C8):14683.

Gerdes, R. (1993b). A primitive equation ocean circulation model using a general vertical coordinate transformation: 2. Application to an overflow problem. Journal of Geophysical Research, 98(C8):14703.

Griffies, S. M. (2004). Fundamentals of Ocean Climate Models. Princeton University Press, Princeton, NJ.

Griffies, S. M., Böning, C., Bryan, F. O., Chassignet, E. P., Gerdes, R., Hasumi, H., Hirst, A., Treguier, A.-M., and Webb, D. (2000a). Developments in ocean climate modelling. Journal of Computational Physics, 2(3-4):123-192.

Griffies, S. M., Pacanowski, R. C., and Hallberg, R. W. (2000b). Spurious Diapycnal Mixing Associated with Advection in a z -Coordinate Ocean Model. Monthly Weather Review, 128(3):538-564.

Haidvogel, D. and Beckmann, A. (1999). Numerical Ocean Circulation Modeling. Imperial College Press.

Haidvogel, D. B., Beckmann, A., and Hedström, K. S. (1991). Dynamical simulations of filament formation and evolution in the Coastal Transition Zone. Journal of Geophysical Research, 96(C8):15017.

Haney, R. (1991). On the pressure gradient force over steep topography in sigma coordinate ocean models. Journal of Physical Oceanography, 21:610-619.

Hirt, C., Amsden, A., and Cook, J. (1974). An arbitrary LagrangianâĂŞEulerian computing method for all flow speeds. Journal of Computational Physics, $14(3): 227-253$.

Hofmeister, R., Burchard, H., and Beckers, J. M. (2010). Non-uniform adaptive vertical grids for 3D numerical ocean models. Ocean Modelling, 33(1-2):70-86.

Holt, J., Hyder, P., Ashworth, M., Harle, J., Hewitt, H. T., Liu, H., New, A. L., Pickles, S., Porter, A., Popova, E., Allen, J. I., Siddorn, J., and Wood, R. (2017). Prospects for improving the representation of coastal and shelf seas in global 
ocean models. Geoscientific Model Development, 10(1):499-523.

9 Ivanov, V. V., Shapiro, G. I., Huthnance, J. M., Aleynik, D. L., and Golovin, P. N. (2004). Cascades of dense water around the world ocean. Progress in Oceanography, 60(1):47-98.

James, I. (1996). Advection schemes for shelf sea models. Journal of Marine Systems, 8(3-4):237-254.

Kantha, L. H. and Clayson, C. A. (2000). Numerical Models of Oceans and Oceanic Processes. Academic Press.

Kasahara, A. (1974). Various Vertical Coordinate Systems Used for Numerical Weather Prediction. Monthly Weather Review, 102(7):509-522.

Klingbeil, K., Mohammadi-Aragh, M., Gräwe, U., and Burchard, H. (2014). Quantification of spurious dissipation and mixing âĂ Discrete variance decay in a Finite-Volume framework. Ocean Modelling, 81:49-64.

Laanaia, N., Wirth, A., Molines, J. M., Barnier, B., and Verron, J. (2010). On the numerical resolution of the bottom layer in simulations of oceanic gravity currents. Ocean Science, 6(2):563-572.

Leclair, M. and Madec, G. (2011). z-Coordinate, an Arbitrary Lagrangian-Eulerian coordinate separating high and low frequency motions. Ocean Modelling, 37(34):139-152.

Legg, S., Briegleb, B., Chang, Y., Chassignet, E. P., Danabasoglu, G., Ezer, T., Gordon, A. L., Griffies, S., Hallberg, R., Jackson, L., Large, W., Özgökmen, T. M., Peters, H., Price, J., Riemenschneider, U., Wu, W., Xu, X., and Yang, J. (2009). Improving Oceanic Overflow Representation in Climate Models: The Gravity Current Entrainment Climate Process Team. Bulletin of the American Meteorological Society, 90(5):657-670.

Legg, S., Hallberg, R. W., and Girton, J. B. (2006). Comparison of entrainment in overflows simulated by z-coordinate, isopycnal and non-hydrostatic models. Ocean Modelling, 11(1-2):69-97.

Lemarié, F., Kurian, J., Shchepetkin, A. F., Jeroen Molemaker, M., Colas, F., and McWilliams, J. C. (2012). Are there inescapable issues prohibiting the use of terrain-following coordinates in climate models? Ocean Modelling, 42:57-79.

Madec, G. (2008). NEMO ocean engine. Note du Pôle de modélisation, Institut Pierre-Simon Laplace (IPSL), (27):357pp.

Madec, G., Delecluse, P., Crépon, M., and Lott, F. (1996). Large-Scale Preconditioning of Deep-Water Formation in the Northwestern Mediterranean Sea. Journal of Physical Oceanography, 26(8):1393-1408.

Maqueda, M. M. and Holloway, G. (2006). Second-order moment advection scheme applied to Arctic Ocean simulation. Ocean Modelling, 14(3-4):197-221.

Marchesiello, P., Debreu, L., and Couvelard, X. (2009). Spurious diapycnal mixing in terrain-following coordinate models: The problem and a solution. Ocean Modelling, 26(3-4):156-169.

Marshall, J. and Schott, F. (1999). Open-ocean convection: Observations, theory, and models. Reviews of Geophysics, 37(1):1-64.

Marti, O., Madec, G., and Delecluse, P. (1992). Comment on 'Net diffusivity in ocean general circulation models with nonuniform grids' by F. L. Yin and I. Y. Fung. Journal of Geophysical Research, 97(C8):12763.

Martinho, A. S. and Batteen, M. L. (2006). On reducing the slope parameter in terrain-following numerical ocean models. Ocean Modelling, 13(2):166-175. 
Mellor, G. L. and Blumberg, A. F. (1985). Modeling Vertical and Horizontal Diffusivities with the Sigma Coordinate System. Monthly Weather Review, 113(8):1379-1383.

Mellor, G. L., Ezer, T., and Oey, L.-Y. (1994). The Pressure Gradient Conundrum of Sigma Coordinate Ocean Models. Journal of Atmospheric and Oceanic Technology, 11(4):1126-1134.

Mellor, G. L., Häkkinen, S. M., Ezer, T., and Patchen, R. C. (2002). A Generalization of a Sigma Coordinate Ocean Model and an Intercomparison of Model Vertical Grids. In Pinardi, N. and Woods, J., editors, Ocean Forecasting: Conceptual Basis and Applications, pages 55-72. Springer Berlin Heidelberg, Berlin, Heidelberg.

Mellor, G. L., Oey, L. Y., and Ezer, T. (1998). Sigma coordinate pressure gradient errors and the seamount Problem. Journal of Atmospheric and Oceanic Technology, 15(5):1122-1131.

MyOcean2 (2014). MyOcean catalogue of PRODUCTS, V4.0.

Nof, D. (1983). The translation of isolated cold eddies on a sloping bottom. Deep Sea Research Part A, Oceanographic Research Papers, 30(2):171-182.

Oddo, P., Adani, M., Pinardi, N., Fratianni, C., Tonani, M., and Pettenuzzo, D. (2009). A nested Atlantic-Mediterranean Sea general circulation model for operational forecasting. Ocean Science, 5:461-473.

O'Dea, E. J., Arnold, A. K., Edwards, K. P., Furner, R., Hyder, P., Martin, M. J., Siddorn, J. R., Storkey, D., While, J., Holt, J. T., and Liu, H. (2012). An operational ocean forecast system incorporating NEMO and SST data assimilation for the tidally driven European North-West shelf. Journal of Operational Oceanography, 5(1):3-17.

Pacanowski, R. C., Gnanadesikan, A., and Olume, V. (1998). Transient Response in a Z -Level Ocean Model That Resolves Topography with Partial Cells. Monthly Weather Review, 126(12):3248-3270.

Redi, M. H. (1982). Oceanic Isopycnal Mixing by Coordinate Rotation. Journal of Physical Oceanography, 12(10):1154-1158.

Roberts, M. and Marshall, D. (1998). Do We Require Adiabatic Dissipation Schemes in Eddy-Resolving Ocean Models? Journal of Physical Oceanography, 28(10):2050-2063.

Roquet, F., Madec, G., Brodeau, L., and Nycander, J. (2015). Defining a Simplified Yet Realistic Equation of State for Seawater. Journal of Physical Oceanography, 45(10):2564-2579.

Shapiro, G. I. and Hill, A. E. (1997). Dynamics of Dense Water Cascades at the Shelf Edge. Journal of Physical Oceanography, 27(11):2381-2394.

Shapiro, G. I., Luneva, M., Pickering, J., and Storkey, D. (2013). The effect of various vertical discretization schemes and horizontal diffusion parameterization on the performance of a 3-D ocean model: the Black Sea case study. Ocean Science, 9(2):377-390.

Shchepetkin, A. F. (2003). A method for computing horizontal pressure-gradient force in an oceanic model with a nonaligned vertical coordinate. Journal of Geophysical Research, 108(C3):3090.

Shchepetkin, A. F. and McWilliams, J. C. (2005). The regional oceanic modeling system (ROMS): a split-explicit, free-surface, topography-following-coordinate oceanic model. Ocean Modelling, 9(4):347-404. 
Siddorn, J. R. and Furner, R. (2013). An analytical stretching function that combines the best attributes of geopotential and terrain-following vertical coordinates. Ocean Modelling, 66:1-13.

Song, Y. and Haidvogel, D. (1994). A Semi-implicit Ocean Circulation Model Using a Generalized Topography-Following Coordinate System. Journal of Computational Physics, 115(1):228-244.

Song, Y. T. and Hou, T. Y. (2006). Parametric vertical coordinate formulation for multiscale, Boussinesq, and non-Boussinesq ocean modeling. Ocean Modelling, 11(3-4):298-332.

Treguier, A. M., Dukowicz, J. K., and Bryan, K. (1996). Properties of nonuniform grids used in ocean general circulation models. Journal of Geophysical Research: Oceans, 101(C9):20877-20881.

Trotta, F., Fenu, E., Pinardi, N., Bruciaferri, D., Giacomelli, L., Federico, I., and Coppini, G. (2016). A Structured and Unstructured grid Relocatable ocean platform for Forecasting (SURF). Deep-Sea Research Part II: Topical Studies in Oceanography, 133:54-75.

Umlauf, L. and Burchard, H. (2003). A generic length-scale equation for geophysical turbulence models. Journal of Marine Research, 61(2):235-265.

Umlauf, L. and Burchard, H. (2005). Second-order turbulence closure models for geophysical boundary layers. A review of recent work. Continental Shelf Research, 25(7-8):795-827.

Valeport (2017). Model 106 Current Meter. http://www.valeport.co.uk/Portals/0/Docs/Datasheets/Valeport-Model106.pdf. Accessed 12 November 2017.

Wobus, F., Shapiro, G. I., Huthnance, J. M., and Maqueda, M. A. M. (2013). The piercing of the Atlantic Layer by an Arctic shelf water cascade in an idealised study inspired by the Storfjorden overflow in Svalbard. Ocean Modelling, 71:5465.

Wobus, F., Shapiro, G. I., Maqueda, M., and Huthnance, J. (2011). Numerical simulations of dense water cascading on a steep slope. Journal of Marine Research, $69(2): 391-415$. 